\title{
Performance of Adaptive Receivers in a
}

\section{Mobile Environment}

\author{
G. Castellini ${ }^{*}$, E. Del Re ${ }^{\circ}$ and L. Pierucci ${ }^{\circ}$ \\ ${ }^{*}$ I.R.O.E. - CNR Via Panciatichi,64 50127 Firenze,Italy \\ - University of Florence, Department of Electronic En- \\ gineering Via S.Marta,3 50139 Firenze, Italy
}

\section{$1 \quad$ Introduction}

Mobile communications are going to be one of the fastest growing fields in the future, due to the high number of users.

The narrow band TDMA digital cellular system has required to adopt channel coding, interleaving and compact-spectrum constant envelope modulation (as the modulations belonging to the class of the Continuous Phase Modulation CPM) to increase the spectrum efficiency. Due to the spectral shape of the adopted partial response modulation and to the multipath nature of the communication channel, interference occurs between adjacent symbols, which are known as inter-symbol interference (ISI). The best theoretical performance for demodulating operations over channels with ISI and additive white noise is the maximum likelihood sequence estimator (MLSE) technique [3]-[5]. Topics about different digital MLSE structures, such as the correlator receiver [5] and the euclidean distance polyphase receiver which can be efficiently implemented by means of the Viterbi algorithm, are analyzed and discussed. An Euclidean MLSE structure leads to a receiver that searches among all possible data sequences to find the sequence which is closest to the noisy received signal according to the Euclidean metric directly used in the Viterbi algorithm. This euclidean MLSE receiver is analyzed in the paper since no matched filter is needed and, in view of adaptive implementations, the Viterbi processor gives directly the error signal used by adaptation algorithms. In the mobile environ- 
ment, the TDMA signal structure and the rapidly varying channel characteristics, due to fading and Doppler effect, require adaptive techniques through a continuous updating of channel characteristics. The channel estimation is usually realized by a FIR transversal filter with adjustable coefficients. Either the gradient LMS algorithm or one of the class of the faster converging RLS algorithm may be used to adjust the coefficients of the filter. These estimated coefficients are fed to the MLSE based on VA for use in the metric computations [6]. This conventional adaptive MLSE receiver [3],[5],[7] has difficulty in tracking fast time-varying ISI channels due to the fixed decision delay inherent in the Viterbi algorithm which causes a channel estimation delay [29].

In the literature [8],[29], the per-survivor processing (PPSP) and the adaptive MLSE proposed by Kubo et alii are presented to embed data-aided estimation techniques without being influenced by the fixed decision delay, within the Viterbi algorithm itself. In these method the estimate of the channel impulse response is evaluated by using the data sequence associated to each survivor path in the branch metric calculation which is relative to a possible state transition in the Viterbi trellis. Therefore for each survivor independent channel coefficients are updated employing conventional adaptive algorithms. The paper presents a new method in which the initial estimate of channel response for each burst is obtained by the known training sequence and the parameters update is subsequently carried out recursively at each step of the Viterbi algorithm, during the unknown information sequence, taking into account the data sequence that corresponds to the best metric value (minimum-survivor method). The selected data sequence at each step is considered to be one of the closest to the received signal and is used to update the channel estimate of all survivors. However the decision on the transmitted data sequence is taken by the Viterbi algorithm at the end of each burst. Our investigation is similar to PPSP but in the final analysis the min survivor method highlights a significantly lower computational complexity with respect to persurvivor principle giving equivalent performance in typical GSM environments as shown in the simulation results. The paper is organized as follows. Section 2 describes the GMSK modulation as belonging to the class of the CPM modulation. Section 3 gives the model of the communication channel including fading and Doppler effects. In Section 4 the structure of different MLSE receivers are analyzed. Section 5 indicates the independence of the proposed MLSE system from carrierphase and symbol-timing synchronization. The classical adaptive algorithms, such as the least mean square (LMS) and the recursive least square (RLS) algorithms used within the Viterbi algorithm to track the rapidly time-variant channel are described in Section 6. Finally, in Section 7 the performance of the MLSE receiver in the two operating modes, periodically or continuously updating, in terms of bit error rate (BER) versus energy per bit/ noise spectral $\operatorname{density}\left(E_{b} / N_{0}\right)$ is shown, and the conclusions can be found in Section 8 . 


\section{Linear model of CPM signals}

In this section we briefly recall the relevant characteristics of Continuous Phase Modulation (CPM) signals, with a modulation index $h=\frac{1}{2}$ and modulated by a sequence of binary alphabet symbols $x_{n}= \pm 1$. Any CPM signal can be defined by the continuous phase shift function $\varphi(t)$. In terms of complex envelope representation, it has the general form [1]

$$
r_{0}(t)=e^{j \pi h \sum_{n} x_{n} \varphi(t-n T)}
$$

where $\mathrm{T}$ is the bit period and $j=\sqrt{-1}$. The phase shift function is assumed to be zero for negative values of time $t$ and has the unit value for $t \geq\left(L_{m}-1\right) T, L_{m}$ being a positive integer. Examples of modulations belonging to this class are MSK (Minimum Shift Keying) and GMSK (Gaussian Minimum Shift Keying) $[1],[2],[9]$. As shown [2], in some cases of interest the $h=\frac{1}{2}$ CPM signal can be very closely approximated by a sum of time and phase shifted pulses as follows

$$
r_{1}(t)=\sum_{n} j^{n} z_{n} c(t-n T) \simeq r_{0}(t)
$$

In eq. $2 z_{n}$ symbols are determined by the recursion

$$
z_{n}=x_{n} z_{n-1}
$$

and the pulse $c(t)$, which approximates the form of the modulation phase shape and has a duration of less than or equal to $L_{m} T$, is defined by

$$
c(t)=c_{0}(t)=\prod_{i=0}^{L_{m}-2} \sin \left[\frac{\pi}{2} \psi(t+i T)\right]
$$

where

$$
\psi(t)= \begin{cases}\varphi(t) & t \leq\left(L_{m}-1\right) T \\ 1-\varphi\left(t-\left(L_{m}-1\right) T\right) & t>\left(L_{m}-1\right) T\end{cases}
$$

The factor $j^{n}$ in 2, that causes $\pi / 2$ phase rotation on the complex plane from symbol to symbol, can be avoided by means of a derotation technique [10], e.g. by multiplying, at the receiver, the signal $r_{1}(t)$ by the complex function: $d(t)=(-j)^{i}$ for $i T \leq t<(i+1) T$. Hence, the signal takes the form

$$
r_{2}(t)=d(t) r_{1}(t)=\sum_{n} z_{n} p(t-n T)
$$

having defined a derotated pulse $p(t)=d(t) c(t)$. Finally, let $a_{n}$ be the assigned binary sequence to be modulated. In order to have $a_{n}$ in the linear representation 5 the transmitter should build the phase in the exponent of 1 after a 
differential encoding of the sequence: from 3 , choosing $x_{n}=a_{n} a_{n-1}$, it results $\left\{z_{n}\right\}=\left\{a_{n}\right\}$.

In this way, a $h=\frac{1}{2}$ CPM modulation can be depicted as a simple binary Pulse Amplitude Modulation (PAM) signal, thus simplifying the signal model and the receiver structure. In the following, for the baseband transmitted signal the linear model

$$
r(t)=\sum_{n} a_{n} p(t-n T)
$$

will be assumed for the baseband signal. Accordingly, the signal modulated onto a carrier frequency $f_{0}$ is

$$
r_{M}(t)=e^{j 2 \pi f_{0} t} \sum_{n} a_{n} p(t-n T)
$$

The parameter $L_{m}$ represents the duration (in symbols) of the modulating pulse $p(t)$ and, consequently, of the controlled intersymbol interference (ISI) inherent to the modulation itself. Typical values of $L_{m}$ are in the range 2 to 4 .

\section{Channel effects on signal}

In land mobile radio, the received signal is subjected to multipath components caused by many factors, including tropospheric scattering, reflections from natural and artificial of objects, topographic and environmental conditions. All these factors lead to characterize the signal amplitude received at the mobile unit as composed of two terms [15]:

- a slow fading component, mainly due to the local topographic conditions, antenna height and other environmental conditions. This slow fading component of the received signal may remain approximately constant along distances of the order of 20 to 30 wavelengths (at least for frequencies below $1 \mathrm{GHz}$ );

- fast fading component, due to the reflections from obstacles and the vehicle speed. This component must be carefully considered in the receiver design. Generally the assumed model for the envelope of these signal affected by this type of fading is the Rayleigh distribution or the Rice distribution. The first one applies when no "dominant" component is present in the received signal. 
In case of digital transmission another parameter is important, the delay spread or the maximum delay. Due to the multipath propagation, a transmitted impulse signal produces several replicas at the receiver at different time instants. The delay spread is defined as the standard deviation of the delay time of the received signal and it measures the time dispersion of the received signal.

In some cases more useful is the delay of the last received significant replica (maximum delay) instead of the delay spread. In the $900 \mathrm{MHz}$ band the delay spread is typically about $0.1 \mu s$ on flat terrain, $2 \mu s$ in urban areas, and up to $5 \mu s$ for hilly terrain. The maximum delay can be $0.5 \mu s, 10 \mu s$ and $20 \mu s$ in the three environments respectively. The fading signal components are received with independently time-varying amplitudes and phases, with random incoming angle and time delay [12],[11],[13]. The channel model is composed of 6-12 distinct propagation paths which are subjected to the Rayleigh fading; each path is characterized by a delay $d_{i}(t)$ and by a specific attenuation $A_{i}(t)$ and the classical Doppler spectrum is employed [14] (fig. 1). The baseband

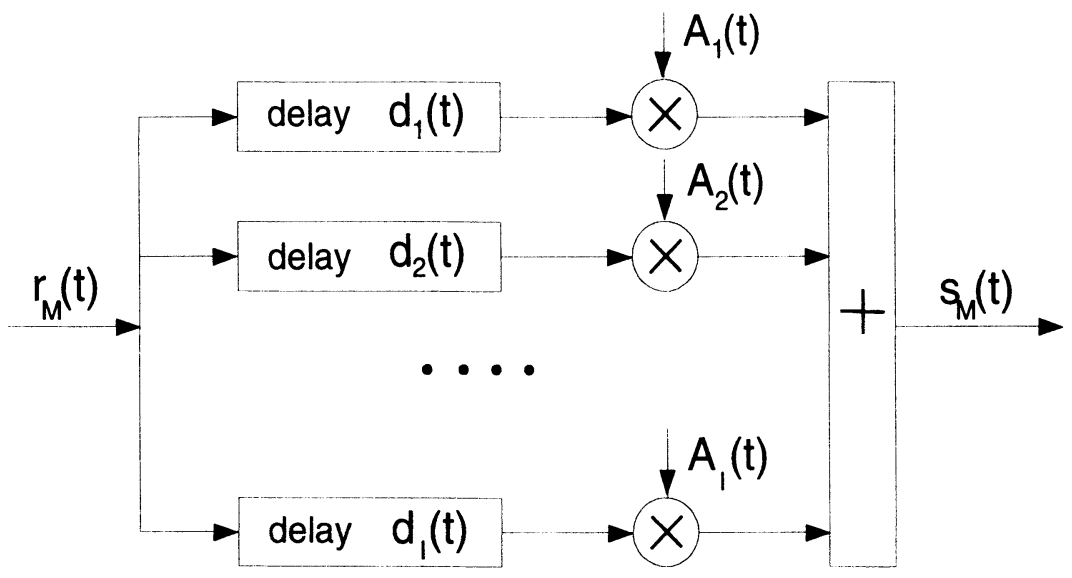

Fig. 1 Mobile radio channel model 
expression for the noiseless received signal is

$$
s(t)=s_{M}(t) e^{-j 2 \pi f_{0} t}=\sum_{n} a_{n} g(t, t-n T)
$$

In the last one (in which $s_{M}(t)$ is the modulated representation of received signal), the received pulse shape $g(t, \tau)$ has the form

$$
g(t, \tau)=\sum_{i=1}^{I} w_{i}(t) p\left(\tau-d_{i}(t)\right)
$$

where

$$
w_{i}(t)=A_{i}(t) e^{-j 2 \pi f_{0} d_{i}(t)}
$$

Therefore, the channel behaves on the signal as a time-variant linear system with impulse response [3]

$$
h(t, \tau)=\sum_{i=1}^{I} w_{i}(t) \delta\left(\tau-d_{i}(t)\right)
$$

where $\delta(\tau)$ is the Dirac impulsive function. The effects of transmitter and receiver bandpass filters on the pulse shape are included in the impulse response of the channel. In the equation 11 the dependence of the impulse response on variable $t$ through the weights $w_{i}(t)$ and the delays $d_{i}(t)$ accounts for the time changes in the channel. The rate of change of the processes $w_{i}(t)$ (due to the large factor $f_{0}$ in the exponent of 10) is much more critical then that of $d_{i}(t)$ which are assumed to be constant for long time intervals. As a rough measure of the bandwidth of the processes $w_{i}(t)$, which is correlated to the fast fading components, the Doppler frequency $f_{d}=\frac{v}{c} f_{0}$, where $\mathrm{v}, \mathrm{c}$ are respectively the vehicle speed and the velocity of light, can be chosen. For example, considering high vehicle speed of $300 \mathrm{~km} / \mathrm{h}$ and a carrier frequency of $900 \mathrm{MHz}$ the peak Doppler shift that may occur is $250 \mathrm{~Hz}$.

In the applications of interest the channel model can be considered constant during a time interval equal to at least some bit periods, since the bit rate $f_{t}=1 / T$ is much greater than the Doppler frequency even for high vehicle speed. The dependence of the impulse response from variable $\tau$ determines instead the shape of the received pulse. Let $L_{C}$ be the duration of $h(\tau, t)$ expressed in units of T. Then the duration of the ISI in the received signal exceeds the quantity $L_{m}$, intrinsic in the modulation itself, up to $L_{S}=L_{m}+L_{C}$. In the system under examination, typical values for $L_{C}$ parameter are 1-2 in rural or urban environments [15]. Let $n(t)$ be the baseband additive noise introduced in the channel, in such a way that the baseband received signal expression is

$$
y(t)=s(t)+n(t)
$$


The additive noise $n(t)$ is supposed to be stationary, complex-gaussian distributed and, for generality, colored with autocorrelation function $k(t)$. As it will be seen, an important quantity for the definition of the MLSE receiver is the noise inverse autocorrelation quantity $k^{-1}(t)$, which is defined by the relation

$$
\int_{-\infty}^{\infty} k(\tau) k^{-1}(t-\tau) d \tau=\delta(t)
$$

and which is assumed to be zero out of some interval $\left[-L_{n} T ; L_{n} T\right]$, being $L_{n}$ a positive integer.

\section{Digital MLSE receivers - the employment of euclidean distance}

In this section, in order to explain the MLSE euclidean distance structure $[3],[18]$, the channel will be assumed time-invariant, that is the received pulse $g(t, \tau)$ is equal to $g(\tau)$ for each $t$.

According to the MLSE principle, the demodulation of digital information is accomplished through sequences and a single final decision is taken in favor of one sequence $\left\{\hat{a}_{n}\right\}$ only at the end, after the whole transmitted signal is received (at the end of a finite-length sequence in GSM).

In order to reach this aim, a likelihood function is defined on the space of possible candidate sequences $\left\{\alpha_{n}\right\}$ which returns, for each sequence, a measure of its probability of having been transmitted. This function depends, in the particular case, on:

- system impulse response;

- power density spectrum of additive noise introduced in the link;

- carrier phase and symbol timing synchronization;

- received signal.

Indicating $s\left(t \mid\left\{\alpha_{n}\right\}\right)=\sum_{n} \alpha_{n} g(t-n T)$ as the analog expected signal which would be received in absence of noise, considering the transmission of $\left\{\alpha_{n}\right\}$ sequence, the a posteriori transmission probability for $\left\{\alpha_{n}\right\}$ is exponentially decreasing with the following quantity [5]:

$$
\int_{-\infty}^{\infty} \int_{-\infty}^{\infty}\left[y^{*}\left(t_{2}\right)-s^{*}\left(t_{2} \mid\left\{\alpha_{n}\right\}\right)\right] k^{-1}\left(t_{2}-t_{1}\right)\left[y\left(t_{1}\right)-s\left(t_{1} \mid\left\{\alpha_{n}\right\}\right)\right] d t_{1} d t_{2}
$$


where $*$ indicates complex conjugate. This expression represents a distance between received and expected signal, conveniently weighed by the autocorrelation properties of additive noise. It is possible to solve recursively the expression 14 defining functions, in terms of sum of partial metrics, still monotone with the a posteriori transmission probability [24],[5]. They are of the form:

$$
F\left(\left\{\alpha_{n}\right\}\right)=\sum_{n=1}^{N} f_{n}\left(\alpha_{n-L+1}, \ldots, \alpha_{n-1}, \alpha_{n}\right)
$$

where $\mathrm{N}$ is the sequence length and $f_{n}$ are the branch metrics (or functions), depending each one on $L$ consecutive bits in the sequence, where $L$ from the literature[24],[5] is the nearest integer greater than the sum of the received pulse duration $L_{S}$ and the noise inverse autocorrelation function semiduration $L_{n}$. In the following it will always be $L=\left\lceil L_{n}+L_{S}\right\rceil$.

In order to identify the ML sequence, due to the particular structure in 15 , it is possible to optimize the function $F\left(\left\{\alpha_{n}\right\}\right)$ with respect to the sequences using the Viterbi algorithm, which is characterized by an only linear complexity (through a factor $2^{L-1}$ ), instead of exponential, with the sequence length $N$ [15].

Furthermore, for a digital implementation of the MLSE receiver, it is convenient to operate with data taken at some sampling frequency $f_{s}=C f_{r}$ (where $f_{r}=1 / T$ is the bit rate and $C$ an integer) sufficiently high according to the modulation bandwidth. Then let $y_{n}=y(n T / C)$ be the sampled received signal.

A classical MLSE receiver structure, based on a correlation function was first proposed by Ungerboek [5]. It consists of a matched filter cascaded by a decimation device that reduces the sampling frequency to the symbol rate $f_{r}$. The output signal $z_{n}$ is then sent to a Viterbi processor to accomplish MLSE (fig. 2 ). The filter is matched to the system impulse response $g(t)$ and to the noise inverse autocorrelation $k^{-1}(t)$. Its equivalent analog impulse response is:

$$
\int_{-\infty}^{\infty} k^{-1}(t) g^{*}(\tau-t) d \tau
$$

The Viterbi branch function evaluates:

$$
f_{1, n}\left(\alpha_{n-L+1}, \ldots, \alpha_{n}\right)=\Re\left\{\alpha_{n}\left(z_{n}-\sum_{i=0}^{L-1} c_{i} \alpha_{n-i}\right)\right\}
$$

where

$$
c_{i}=\left(1-\frac{\delta_{i}}{2}\right) \int_{-\infty}^{\infty} \int_{-\infty}^{\infty} g^{*}\left(t_{2}\right) k^{-1}\left(t_{2}-t_{1}\right) g\left(t_{1}-i T\right) d t_{1} d t_{2} \quad \delta_{i}= \begin{cases}1 & i=0 \\ 0 & i \neq 0\end{cases}
$$




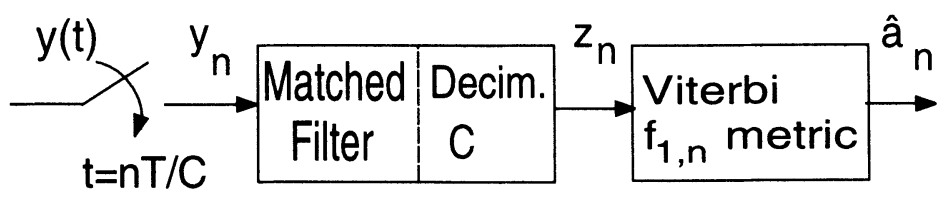

Fig. 2 Correlation MLSE receiver model

and

$$
z_{n}=\int_{-\infty}^{\infty} \int_{-\infty}^{\infty} g^{*}\left(t_{2}-n T\right) k^{-1}\left(t_{2}-t_{1}\right) y\left(t_{1}\right) d t_{1} d t_{2}
$$

and can be interpreted as a correlation in the sampled domain. The structure of the MLSE receivers commonly employed (i.e., see [16],[17]) is based on this correlation operation.

Another possibility of implementation of the MLSE principle is based on euclidean distance. Let $q(t)$ be a complex valued function such that

$$
\int_{-\infty}^{\infty} q(\tau) q^{*}(\tau-t) d \tau=k^{-1}(t)
$$

The function $q(t)$ represents a spectral factorization of the noise inverse autocorrelation $k^{-1}(t)$ (in the frequency domain the function $Q(f)$ is such that $\left.|Q(f)|^{2}=K^{-1}(f)\right)$ and can be chosen with a duration, in the sampled domain, equal to half the duration of $k^{-1}(t)$. Moreover a filter with impulse response $q(t)$ is a whitening filter for the signal $y(t)$. Substituting the equation 19 in the equation 14 yields

$$
\int_{-\infty}^{\infty}\left|z(t)-\sum_{n} \alpha_{n} c(t-n T)\right|^{2} d t
$$

where

$$
c(t)=\int_{-\infty}^{\infty} g(\tau) q(t-\tau) d \tau
$$




$$
z(t)=\int_{-\infty}^{\infty} y(\tau) q(t-\tau) d \tau=\sum_{n} a_{n} c(t-n T)+\int_{-\infty}^{\infty} n(\tau) q(t-\tau) d \tau
$$

According to the last equation the $z(t)$ signal represents a linear modulation with pulse shape $c(t)$ plus AWGN. The quantity 20 can be evaluated in the sampled domain and used directly for the definition of a likelihood function. Assuming the notations

$$
\begin{gathered}
c^{(k)}(i)=c\left(i T+k \frac{T}{C}\right) \quad i=0, \ldots, L-1 \quad k=0, \ldots, C-1 \\
z_{n}^{(k)}(i)=z\left(n T+k \frac{T}{C}\right) \quad n=1, \ldots, N \quad k=0, \ldots, C-1
\end{gathered}
$$

the Viterbi euclidean distance branch function can be defined as follows:

$$
f_{2, n}\left(\alpha_{n-L+1}, \ldots, \alpha_{n}\right)=\sum_{k=0}^{C-1} f_{n}^{(k)}=\sum_{k=0}^{C-1}\left|z_{n}^{(k)}-\sum_{i=0}^{L-1} c^{(k)}(i) \alpha_{n-i}\right|^{2}
$$

Fig. 3 shows the euclidean distance MLSE receiver structure. It is noteworthy that the Viterbi algorithm can be implemented in such a processor that contains $\mathrm{C}$ modules that operates in parallel in a polyphase mode.

If the communication link is regarded as a linear discrete time finite impulse response (FIR) system whose input is the information sequence $a_{n}$ and whose output is the noise- whitened signal $z_{n}(k)$, it is found out that the $c^{(k)}(i)$ branch function parameters are the FIR coefficients. Thus, both 20 in the analog domain and 25 in the discrete domain, state the principle of the minimization the euclidean distance between the whitened received signal and the corresponding expected one. Theoretically, when the system impulse response, noise autocorrelation, synchronization constants are deterministic and known at the receiver the employment of the two different MLSE methods 17 and 25 in demodulation determines identical performance because the functions, being monotone with the same quantity, both describe the same likelihood law.

This statement was verified by means of simulations on MSK signaling reception in AWGN channel: both the demodulation methods always return identical ML sequences. Table 1,2 report the BER values obtained from simulation using two different filters and varying the sampling frequency (parameter $\mathrm{C}$ ). It is noteworthy that a value of $\mathrm{C}=2$, that is $f_{s}=2 f_{r}$ is sufficient not to degrade the system performance. This occurs since the bandwidth of MSK signaling is not so wide to require greater receiver sampling frequency. Generally the computational complexity of the euclidean distance structure is higher, as the Viterbi processor must operate on $\mathrm{C}$ branches. Nevertheless the euclidean distance receiver offers some advantages: 


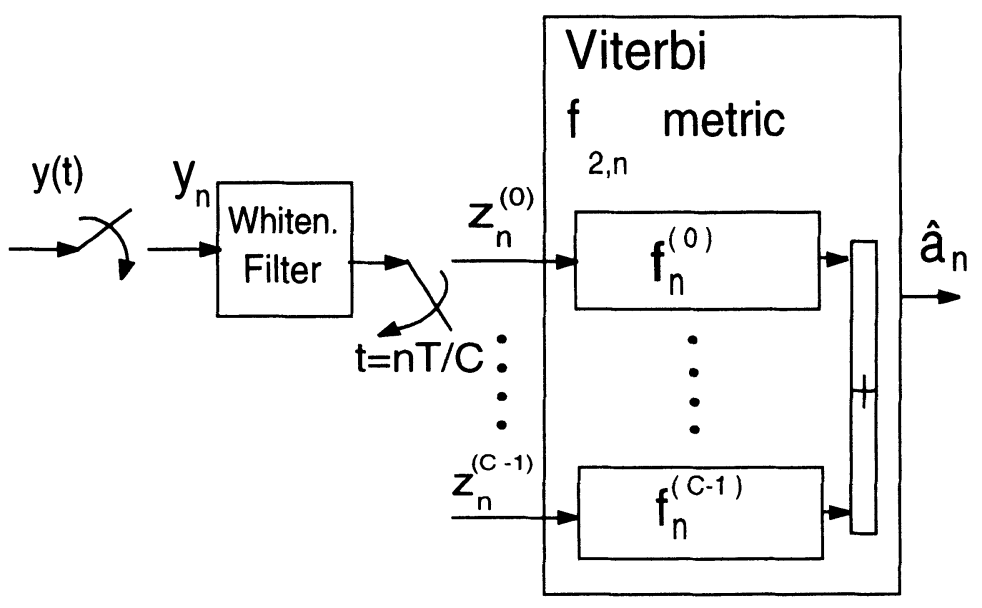

Fig. 3 Euclidean distance MLSE receiver model

- No matched filtering is needed; moreover the whitening filter can be omitted in the practical cases in which the noise in the channel can be assumed to be white

- In applications to narrow-band signaling (receiver sampling rate equal to bit rate: $\mathrm{C}=1$ ) the Viterbi processor consists of only one phase

- In view of adaptive implementations, the number of parameters depending on both $g(t)$ and $k^{-1}(t)$ reduces from $(C+1) L_{s}+(2 C+1) L_{n}$ to $C L_{s}+2 C L_{n}$. When no adaptation to $k^{-1}(t)$ is required, only the $C\left(L_{s}+L_{n}\right)$ Viterbi processor parameters need to be estimated. Furthermore the Viterbi processor directly provides $a_{n}$ error signal directly employable by adaptation algorithms. 


\begin{tabular}{||c||c||c||c||}
\hline \multicolumn{1}{|c||}{ BER } & \multicolumn{3}{|c||}{$E_{b} / N_{0}(d B)$} \\
\cline { 2 - 4 } & 3 & 5 & 8 \\
\hline \hline $8 f_{r}$ & $2.2610^{-2}$ & $5.810^{-3}$ & $1.810^{-4}$ \\
\hline $4 f_{r}$ & $2.3110^{-2}$ & $6.210^{-3}$ & $1.510^{-4}$ \\
\hline $2 f_{r}$ & $2.2910^{-2}$ & $6.310^{-3}$ & $1.710^{-4}$ \\
\hline$f_{r}$ & $2.6610^{-2}$ & $7.410^{-3}$ & $2.810^{-4}$ \\
\hline
\end{tabular}

Table 1 BER performance of euclidean distance and correlation MLSE - MSK signal, AWGN channel, receiver ideal bandpass filters

\begin{tabular}{||c||c||c||c||}
\hline \multicolumn{1}{|c||}{ BER } & \multicolumn{3}{c||}{$E_{b} / N_{0}(d B)$} \\
\cline { 2 - 4 } & 3 & 5 & 8 \\
\hline \hline $8 f_{r}$ & $2.2710^{-2}$ & $5.710^{-3}$ & $1.710^{-4}$ \\
\hline $4 f_{r}$ & $2.2810^{-2}$ & $6.010^{-3}$ & $1.810^{-4}$ \\
\hline $2 f_{r}$ & $2.3110^{-2}$ & $5.810^{-3}$ & $2.010^{-4}$ \\
\hline$f_{r}$ & $2.4710^{-2}$ & $6.610^{-3}$ & $2.610^{-4}$ \\
\hline
\end{tabular}

Table 2 BER performance of euclidean distance and correlation MLSE, MSK signal,AWGN channel, receiver raised cosine filters 


\section{Independence of euclidean MLSE receiver performance from carrier phase and symbol timing synchronization}

In view of the applications it will be assumed hereafter the additive noise to be white and consequently the whitening filter in the euclidean distance receiver is not necessary. As already noticed in the preceding section, the correct likelihood function to be employed in order to obtain MLSE depends on the synchronization, particularly on (fig. 4):

- the phase shift $\theta \in[0,2 \pi)$ between transmitted and local carriers (carrier phase);

- the exact position $\tau \in[0, T / C)$ in which the receiver takes the samples of received signal inside the sampling step (symbol timing).

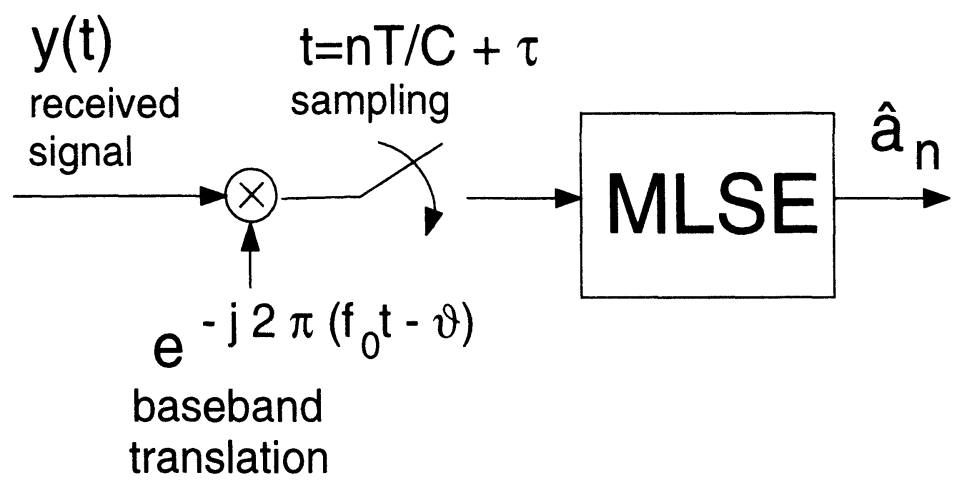

Fig. 4 Carrier phase and symbol timing synchronization parameters

For the euclidean distance MLSE receiver, these quantities determine the values of the coefficients that define the likelihood branch function 25 according to the following relation:

$$
c^{(k)}(i)=e^{j \theta} g\left(\tau+i T+k \frac{T}{C}\right) \quad i=0, \ldots, L-1 \quad k=0, . ., k-1
$$

In particular, as the coefficients are samples of the received pulse $g(t)$, the effect of phase shift is to rotate it on the complex plane while the effect of symbol 
timing is to translate it with respect to $t$. Nevertheless it is possible to show that if the coefficients are correctly defined according to 25 , the probability of error for the euclidean distance based MLSE receiver model is independent from the particular synchronization. This occurs since the euclidean distance between expected and received signals does not depend on complex plan rotations and time translations of both signals.

It is possible to prove that the Euclidean distance between sample sequences received without AWGN and due to two different transmission sequences, $\alpha_{n}(i)$, $\alpha_{n}(j)$, is independent from phase and timing synchronism if their contribution is the same:

$$
d^{2}\left(s\left(\alpha_{n}^{(i)}, \theta, \tau\right), s\left(\alpha_{n}^{(j)}, \theta, \tau\right)\right)=d_{i, j}^{2}
$$

The baseband received signal for every transmitted sequence $\alpha_{n}$ is

$$
s\left(t \mid\left\{\alpha_{n}\right\}=e^{j \theta} \sum_{n} a_{n} g(t-n T)\right.
$$

For example, considering the Euclidean distance for each $\alpha_{n}(i), \alpha_{n}(j)$ and for every and $\tau$, it is obtained

$$
\int\left|\sum\left(\alpha_{n}-\alpha_{j}\right) g(t-n T)\right|^{2} d t
$$

and therefore, the Euclidean distances effectively independent to phase shifts. Analogously for the timing synchronism. Hence in order not to degrade the receiver performance it is sufficient to use the values of $\theta$ and $\tau$ determined by the demodulation and sampling devices in the definition of the branch function. The statement has also been verified by means of computer simulations on MSK signaling in AWGN channel. Table 3 depicts the results. It is recognized that the BER performance does not vary significantly with synchronization. The variations of Table 3 are within the confidence limits of the performed simulations. Then, as an important practical consequence, the employment of a separate subsystem to recover phase and timing synchronization results no longer necessary [18] for MSK signaling. In more general modulation schemes and in case of ISI channel the accuracy of the time synchronization must be within one $\mathrm{T}$ not to lengthening the computing of the metric.

\section{Adaptive euclidean distance MLSE receivers}

In this section, a new adaptive technique named "minimum-survivor" [19],[20] is proposed and compared with per-survivor technique [8]. Among the adaptive 


\begin{tabular}{||l||l||l||l||l||}
\hline \multicolumn{2}{c|}{ BER } & \multicolumn{3}{c||}{$E_{b} / N_{0}(d B)$} \\
\cline { 3 - 5 } \multicolumn{1}{||}{} & & 3 & 5 & 8 \\
\hline \hline$\tau=0$ & $\theta=0^{\circ}$ & $2.6910^{-2}$ & $7.810^{-3}$ & $2.810^{-4}$ \\
\cline { 2 - 5 }$\tau=0$ & $\theta=40^{\circ}$ & $2.6610^{-2}$ & $7.410^{-3}$ & $2.810^{-4}$ \\
\hline$\tau=0$ & $\theta=110^{\circ}$ & $2.6210^{-2}$ & $7.110^{-3}$ & $2.410^{-4}$ \\
\hline$\tau=1 / 2 f_{c}$ & $\theta=0^{\circ}$ & $2.6510^{-2}$ & $7.210^{-3}$ & $3.010^{-4}$ \\
\hline
\end{tabular}

Table 3 BER performance of the euclidean MLSE - MSK signal, AWGN channel, receiver raised cosine filter and $f_{s}=2 f_{r}$

realizations using the LMS and RLS methods, the different minsurvivor and persurvivor estimation techniques have all exhibited very similar performance. Consequently, the simplest structure, which is the proposed LMS min-survivor receiver can be regarded with particular interest and shown in this section. The need to introduce adaptive techniques within the euclidean distance MLSE receiver arises since the likelihood branch function parameters and the correlator receiver depend on the time varying channel characteristics. Only in case of invariant time channel it is possible to measure its impulse response, allowing the receiver to be correctly initialized and permanently operate as a MLSE estimator. In the case of a land mobile radio link, the channel and the synchronization are always unknown and time-varying. Many land mobile communications systems employ the periodic transmission of some established short sequences (preamble) of symbols known by the receiver in order to give start-up values of the likelihood parameters (training processing). Furthermore, the parameters update can be subsequently carried out iteratively throughout the information sequence transmission by means of adaptive algorithms (tracking processing). The employment of tracking techniques is justified by:

1. The training estimation is affected by noise and can be improved by further adaptive estimate.

2. The training estimation needs to be adjusted during the information bits according to the link parameter time changes imposed by fading [21], Doppler effect and any incoherence phenomena.

The conventional adaptive MLSE receiver generally consists of an MLSE estimator implemented by the VA and a channel estimator [29], [7], [5]. 
- the VA evaluates the closest sequence to the transmitted sequence using the channel coefficients supplied by the channel estimator

- the channel estimator adaptively estimates the channel impulse response using the tentative decisions provided by VA as an estimate of the transmitted sequence.

The tentative decisions derived in the VA are obtained with a delay; if the delay is small, erroneous data decisions are given while a large delay causes a channel estimation delay decreasing the capability of tracking fast time-varying ISI channels [29]. Generally, the tentative decisions are made by truncating the survivor path history in the VA to some fixed length usually equal to 5L (L indicates the memory length) giving a channel estimation delay.

As suggested by Qureshi the channel estimation is obtained by using the tentative sequence with the largest survivor metric [5].

In the literature, two similar methods, the persurvivor method [8] and the channel estimation procedure proposed by Kubo et alii [29], estimate the channel impulse response into the structure of the VA itself, along the survivor path connected to each state without the decision delay.

In our method the channel estimation is made directly within the metric calculation of the VA with very restricted delay. The branch metric of the VA calculates the Euclidean distance between the received sequence and the estimate of received signal, therefore the VA directly provides the error signal used by the adaptive algorithms.

Let the time-invariant channel hypothesis be removed and assume that the signal is sampled once per symbol $(\mathrm{C}=1)$. This condition, together with the white-noise condition, leads the euclidean distance MLSE branch function 25 to become

$$
f_{n}=\left|y_{n}-\sum_{i=0}^{L-1} c_{n}(i) \alpha_{n-i}\right|^{2}
$$

where $y_{n}=z_{n}^{(0)}$ is the received signal and $c_{n}(i)=c_{n}^{(0)}(i)$ are the branch function coefficients in which the introduction of dependence on time index $n$ accounts for their time changes. The corresponding monophase MLSE receiver structure is depicted in fig. 5. The aim of adaptivity is to provide the best possible estimation of $c_{n}(i)$ parameters to the Viterbi processor. Since $c_{n}(i)$ are the coefficients of the time-varying FIR that models the communication link, tracking consists particularly in some procedure for the identification of an unknown time varying FIR by observing on the flow of its input and output signals. One estimation problem occurs because while the output signal 


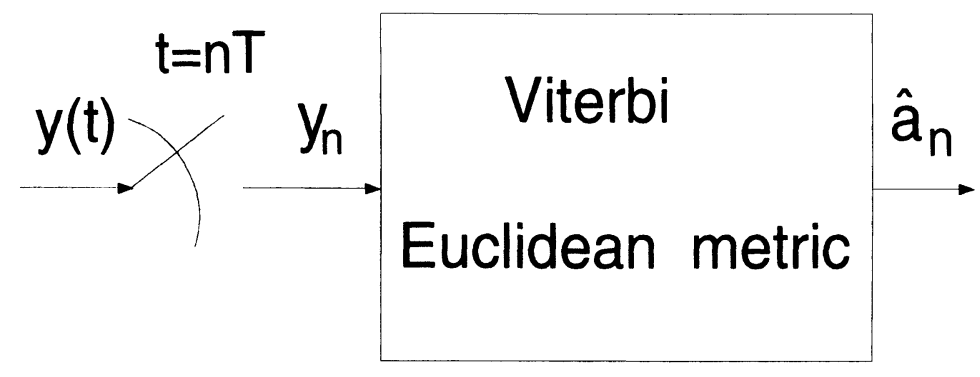

Fig. 5 Mono-phase euclidean distance MLSE receiver model for AWGN signal

$y_{n}$ is available, the $\left\{a_{n}\right\}$ input sequence is unknown and is going to have to be approximated through information supplied by the Viterbi processor itself. Let $\mathbf{c}_{\mathbf{n}}=\left[c_{n}(0), c_{n}(1), . ., c_{n}(L-1)\right]^{T}$ be now the vector of coefficients at time $n$, $\hat{\mathbf{c}}_{\mathbf{n}}=\left[\hat{c}_{n}(0), \hat{c}_{n}(1), . ., \hat{c}_{n}(L-1)\right]^{T}$ its estimation, $\alpha_{\mathbf{n}}=\left[\alpha_{n}, \alpha_{n-1}, . ., \alpha_{n-L+1}\right]^{T}$ a possible transmitted L-uple, $\mathbf{a}_{\mathbf{n}}=\left[a_{n}, a_{n-1}, . ., a_{n-L+1}\right]^{T}$ the actually transmitted one ( $\mathrm{T}$ denotes transpose conjugate). Most system identification methods are based on the criterion of the minimization of the mean square output error $E\left|e_{n}\left(\mathbf{a}_{\mathbf{n}}, \hat{\mathbf{c}}_{\mathbf{n}}\right)\right|^{2}$ with respect to $\hat{\mathbf{c}}_{\mathbf{n}}[22]$, where

$$
e_{n}\left(\mathbf{a}_{\mathbf{n}}, \hat{\mathbf{c}}_{\mathbf{n}}\right)=y_{n}-\hat{\mathbf{c}}_{\mathbf{n}}^{\mathbf{T}} \alpha_{\mathbf{n}}
$$

is the output error, depending on $\hat{\mathbf{c}}_{\mathbf{n}}$. The branch function 30 , generalized as a function of $\hat{\mathbf{c}}_{\mathbf{n}}$ coefficients vector, takes the form

$$
f_{n}\left(\alpha_{\mathbf{n}}, \hat{\mathbf{c}}_{\mathbf{n}}\right)=\left|y_{n}-\hat{\mathbf{c}}_{\mathbf{n}} \alpha_{\mathbf{n}}\right|^{2}=\left|e_{n}\left(\alpha_{\mathbf{n}}, \hat{\mathbf{c}}_{\mathbf{n}}\right)\right|^{2}
$$

As a matter of fact, both the branch function and the mean square output error (to be minimized) are quadratic function of the error. The utility of this functions is in the Gaussian distributed structure of additive noise. Using the euclidean distance adaptive MLSE approach some computational complexity can be avoided; the metric is computed by the Viterbi processor is employed in the tracking algorithm as well. A simple classical adaptive algorithm is the LMS (Least Mean Square) [3],[22],[7] which operates by moving at every time $n$ in the direction of the $\left|e_{n}\left(\mathbf{a}_{\mathbf{n}}, \hat{\mathbf{c}}_{\mathbf{n}}\right)\right|^{2}$ gradient (versus $\mathbf{c}_{\mathbf{n}}$ ). This results in the following iteration:

$$
\hat{\mathbf{c}}_{\mathbf{n}+1}=\hat{\mathbf{c}}_{\mathbf{n}}+\mu e_{n}^{*}\left(\mathbf{a}_{\mathbf{n}}, \hat{\mathbf{c}}_{\mathbf{n}}\right) \mathbf{a}_{\mathbf{n}}
$$


where $\mu$ is the step-size parameter which controls the rate of adjustment. In the particular case, if the $\mathbf{c}_{\mathbf{n}}$ vector are time invariant, the algorithm converges for $\mu<2 / L$ but while the convergence is slower for smaller values of $\mu$, the difference between the residual error and the minimum value of the squared errors grows linearly with $\mu$ through a factor $\sigma^{2} L / 2$, where $\sigma^{2}$ is the additive noise variance. Then, in theory, $\mu$ must be chosen as a compromise between the speed of response and the stability of the algorithm. In the results, the optimization of $\mu$ has been performed by computer simulation for the different system environments.

The RLS (Recursive Least Square) [3],[22],[7] algorithm attains instead the minimization of the function

$$
\sum_{i=0}^{n} w^{n-i}\left|e_{i}\left(\mathbf{a}_{\mathbf{i}}, \hat{\mathbf{c}}_{\mathbf{n}}\right)\right|^{2}=\sum_{i=0}^{n} w^{n-i}\left|y_{i}-\hat{\mathbf{c}}_{\mathbf{n}} \mathbf{a}_{\mathbf{i}}\right|^{2}
$$

where $w \in(0,1)$ is the "forgetting factor" weighing the past output errors according to an exponentially decreasing progression. In the time-invariant taps case, if the input is stationary, the RLS algorithm is asintotically optimal for $w \simeq 1$ since it approximates the minimization of mean square output error law. Nevertheless when the $\mathbf{c}_{\mathbf{n}}$ vector is time-varying a forgetting factor must be introduced by reducing $w$. The $\hat{\mathbf{c}}_{\mathbf{n}}$ computation can be efficiently performed iteratively as follows [3],[30]:

$$
\hat{\mathbf{c}}_{\mathbf{n}+1}=\hat{\mathbf{c}}_{\mathbf{n}}+e_{n}^{*}\left(\mathbf{a}_{\mathbf{n}}, \hat{\mathbf{c}}_{\mathbf{n}}\right) \mathbf{k}_{\mathbf{n}}
$$

where

$$
\mathbf{k}_{\mathbf{n}}=\frac{\mathbf{P}_{\mathbf{n}-\mathbf{1}} \mathbf{a}_{\mathbf{n}}}{w+\mathbf{a}_{\mathbf{n}}^{\mathrm{T}} \mathbf{P}_{\mathbf{n}-\mathbf{1}} \mathbf{a}_{\mathbf{n}}}
$$

is the Kalman weighing vector, and

$$
\mathbf{P}_{\mathbf{n}}=\frac{1}{w}\left(\mathbf{P}_{\mathbf{n}-1}-\mathbf{k}_{\mathbf{n}} \mathbf{a}_{\mathbf{n}}^{\mathbf{T}} \mathbf{P}_{\mathbf{n}-\mathbf{1}}\right)
$$

is the input inverse autocorrelation matrix. The last one must be updated and stored in memory for use at the following step. Both the LMS and RLS algorithms update the $\hat{\mathbf{c}}_{\mathbf{n}}$ by iteratively adding an adjustment term. The adjustment term is given by a vector of weights, which, multiplied by the error $e_{n}$, determines the parameters change. While the increase is forced in the $\mathbf{a}_{\mathbf{n}}$ vector direction in the LMS algorithm and evaluates $\mu e_{n}$ (in absolute value) for each component, in the RLS algorithm the increase in one component is determined by the associated weight in the Kalman gain vector. The LMS and RLS algorithms update the channel coefficients within the VA and therefore the error (or the branch metric calculation) also depends on the state transition. 
The updating of the channel coefficients at time $n+1$ in the Viterbi algorithm is based on the old least squared estimate of the tap-weight vector which was made at time $n$.

Since the sequence $\left\{a_{n}\right\}$ is not available, the LMS and RLS algorithms must use an estimate. In order for the receiver to operate in real-time, the estimation of $\left\{a_{n}\right\}$ cannot supplied by the tentative decisions with fixed delay, but must be continuously updated from the information given by the Viterbi processor. In principle, the idea is to let the Viterbi processor operate in concurrence with the coefficients estimation processor, in such a way that each one at every step receives information from the other one, computes certain quantities, and returns them back to the other one, as in fig. 6. The Viterbi processor calculates the errors, the metrics, the survivor paths, while the other processor estimates the likelihood function parameters. At each step $n$ the set of Viterbi survivor

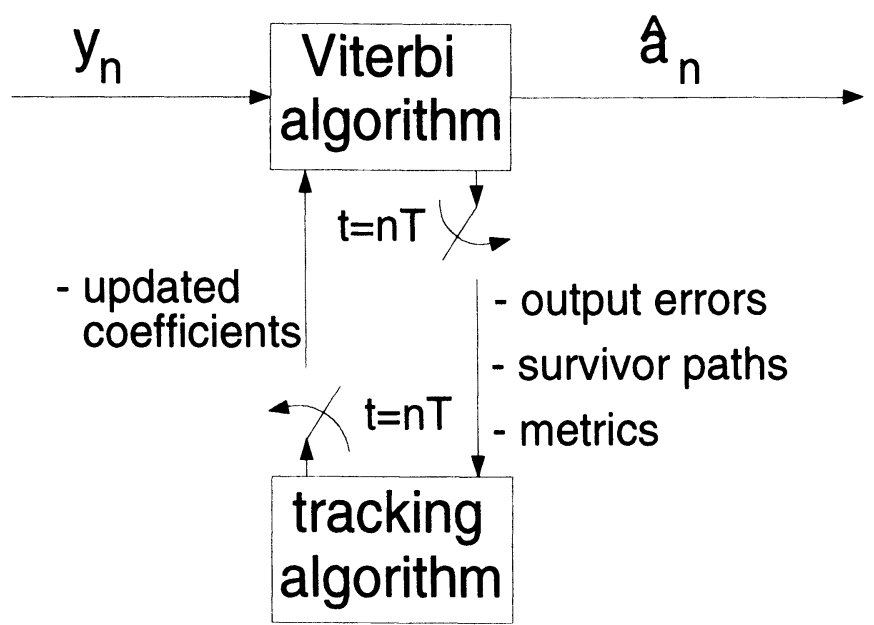

Fig. 6 Concurrent processing of Viterbi algorithm and tracking algoritm

paths includes the only path thereafter candidates to the maximum likelihood estimate. Among them, the one with the best metric value (minimum-survivor, briefly min- survivor) can be regarded as the most likely to be conditioned to the signal samples received and processed till that moment. The realization of minimum-survivor principle is quite simple since it only requires the comparison of all the survivor metrics at every step. The sequence with the largest metric is used to update the branch function coefficients to all the states of the VA. Let $\left\{\hat{a}_{0}(n), \hat{a}_{1}(n), . ., \hat{a}_{n}(n)\right\}$ be the min-survivor at time $n$, which is 
also the instantaneous maximum likelihood sequence at time $n$. It has been verified that the error rate in the above sequence is not uniform with respect to $n$, but can be significantly higher for values close to $n$ [24],[25]; in other words, the survivor paths converge in the past. Thus, in order to approximate within the least errors the true transmitted sequence, it can be convenient to introduce some delay $d>0$ in the symbols $a_{n}$ of the recursions $33,35,37$. Of course, if $d$ chosen is too large, the delay introduced in the estimation process can determine significant performance degradation.

The same formulas become respectively:

$$
\hat{\mathbf{c}}_{\mathbf{n}+\mathbf{1}}=\hat{\mathbf{c}}_{\mathbf{n}}+\mu e_{n-d}^{*}\left(\hat{\mathbf{a}}_{\mathbf{n}-\mathbf{d}}(\mathbf{n}), \hat{\mathbf{c}}_{\mathbf{n}}\right) \hat{\mathbf{a}}_{\mathbf{n}-\mathbf{d}}(\mathbf{n})
$$

for LMS algorithm, and for RLS algorithm

$$
\begin{gathered}
\hat{\mathbf{c}}_{\mathbf{n}+\mathbf{1}}=\hat{\mathbf{c}}_{\mathbf{n}}+\mathbf{e}_{\mathbf{n}-\mathbf{d}}^{*}\left(\hat{\mathbf{a}}_{\mathbf{n}-\mathbf{d}}(\mathbf{n}), \hat{\mathbf{c}}_{\mathbf{n}}\right) \hat{\mathbf{k}}_{\mathbf{n}-\mathbf{d}}(\mathbf{n}) \\
\hat{\mathbf{k}}_{\mathbf{n}-\mathbf{d}}(\mathbf{n})=\frac{\hat{\mathbf{P}}_{\mathbf{n}-\mathbf{d}-\mathbf{1}}(\mathbf{n}-\mathbf{1}) \hat{\mathbf{a}}_{\mathbf{n}-\mathbf{d}}(\mathbf{n})}{w+\hat{\mathbf{a}}_{\mathbf{n}-\mathbf{d}}(\mathbf{n})^{\mathbf{T}} \hat{\mathbf{P}}_{\mathbf{n}-\mathbf{d}-\mathbf{1}}(\mathbf{n}-\mathbf{1}) \hat{\mathbf{a}}_{\mathbf{n}-\mathbf{d}}(\mathbf{n})} \\
\hat{\mathbf{P}}_{\mathbf{n - d}}(\mathbf{n})=\frac{1}{w}\left(\hat{\mathbf{P}}_{\mathbf{n}-\mathbf{d}-\mathbf{1}}(\mathbf{n}-\mathbf{1})-\hat{\mathbf{k}}_{\mathbf{n}-\mathbf{d}}(\mathbf{n}) \hat{\mathbf{a}}_{\mathbf{n}-\mathbf{d}}(\mathbf{n})^{\mathbf{T}} \hat{\mathbf{P}}_{\mathbf{n}-\mathbf{d}-\mathbf{1}}(\mathbf{n}-\mathbf{1})\right)
\end{gathered}
$$

An alternative to the above mentioned method is to accomplish an independent likelihood function coefficients estimation for each survivor (per-survivor processing) [8] At each step in order to calculate the branch metric relative to a possible state transition, the coefficients associated to the source state are employed. Then, after the new set of survivor paths is determined and the corresponding correct (surviving) state transitions are found, for each state transition a coefficients vector updating is carried out. The coefficients associated to the incoming state are updated in terms of:

- the coefficients associated to the source state;

- an adjustment increase depending on the error

- the binary L-uple corresponding to the state transition.

Let us consider a certain state $k_{3}$ at time $n$ which allows the transitions $k_{1} \rightarrow k_{3}$ and $k_{2} \rightarrow k_{3}$ (fig. 7). Let $\hat{\mathbf{c}}_{\mathbf{n}}(k)$ be the estimated coefficients vector associated to the state $k, \alpha\left(k_{i} \rightarrow k_{j}\right)$ the bit L-uple corresponding to the $k_{i} \rightarrow k_{j}$ transition. First, the errors $e_{n}\left(\alpha\left(k_{1} \rightarrow k_{3}\right), \hat{\mathbf{c}}_{\mathbf{n}}(k 1)\right), e_{n}\left(\alpha\left(k_{2} \rightarrow k_{3}\right), \hat{\mathbf{c}}_{\mathbf{n}}\left(k_{2}\right)\right)$ are computed. Then, their squared absolute values (the branch metrics) are computed. By means of the branch metrics, the partial metrics up till time $n$ are 


\section{time: $n-1 \quad n$}

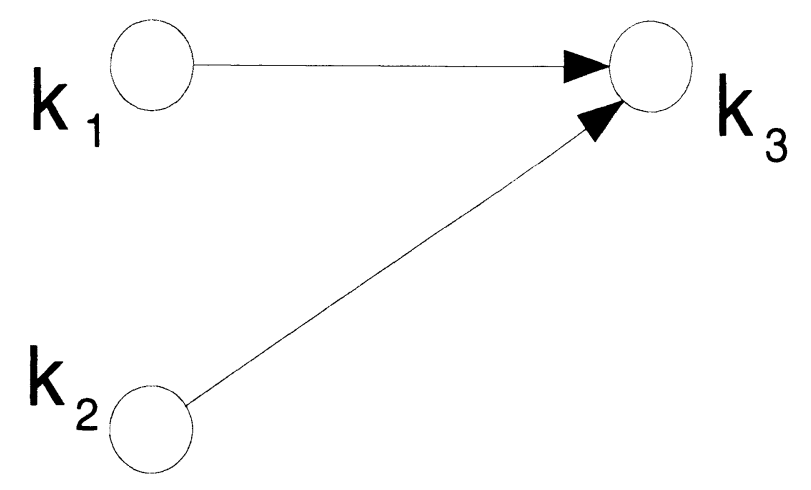

Fig. 7

found, and the survivor at time $n$ is chosen (say the one coming from state $k_{1}$ ). The state transition corresponding to the survivor is then $k_{1} \rightarrow k_{3}$ : now the coefficients associated to state $k_{3}$ can be found. If the LMS algorithm is employed:

$$
\hat{\mathbf{c}}_{\mathbf{n}+\mathbf{1}}\left(k_{3}\right)=\hat{\mathbf{c}}_{\mathbf{n}}\left(k_{1}\right)+\mu e_{n}^{*}\left(\alpha\left(k_{1} \rightarrow k_{3}\right), \hat{\mathbf{c}}_{\mathbf{n}}\left(\mathbf{k}_{\mathbf{1}}\right)\right) \alpha\left(\mathbf{k}_{\mathbf{1}} \rightarrow \mathbf{k}_{\mathbf{3}}\right)
$$

If the RLS algorithm is employed:

$$
\begin{gathered}
\hat{\mathbf{c}}_{\mathbf{n}+\mathbf{1}}\left(k_{3}\right)=\hat{\mathbf{c}}_{\mathbf{n}}\left(k_{1}\right)+e_{n}^{*}\left(\alpha\left(k_{1} \rightarrow k_{3}\right), \hat{\mathbf{c}}_{\mathbf{n}}\left(\mathbf{k}_{\mathbf{1}}\right)\right) \hat{\mathbf{k}}_{\mathbf{n}}\left(\mathbf{n}, \mathbf{k}_{\mathbf{3}}\right) \\
\hat{\mathbf{k}}_{\mathbf{n}}\left(n, k_{3}\right)=\frac{\hat{\mathbf{P}}_{\mathbf{n}-\mathbf{1}}\left(\mathbf{n}-\mathbf{1}, \mathbf{k}_{\mathbf{1}}\right) \alpha\left(\mathbf{k}_{\mathbf{1}} \rightarrow \mathbf{k}_{\mathbf{3}}\right)}{w+\alpha^{\mathrm{T}}\left(\mathbf{k}_{\mathbf{1}} \rightarrow \mathbf{k}_{\mathbf{3}}\right) \hat{\mathbf{P}}_{\mathbf{n}-\mathbf{1}}\left(\mathbf{n}-\mathbf{1}, \mathbf{k}_{\mathbf{1}}\right) \alpha\left(\mathbf{k}_{\mathbf{1}} \rightarrow \mathbf{k}_{\mathbf{3}}\right)} \\
\hat{\mathbf{P}}_{\mathbf{n}}\left(\mathbf{n}, \mathbf{k}_{\mathbf{3}}\right)=\frac{1}{w}\left(\hat{\mathbf{P}}_{\mathbf{n}-\mathbf{1}}\left(\mathbf{n}-\mathbf{1}, \mathbf{k}_{\mathbf{1}}\right)-\hat{\mathbf{k}}_{\mathbf{n}}\left(\mathbf{n}, \mathbf{k}_{\mathbf{3}}\right) \alpha^{\mathrm{T}}\left(\mathbf{k}_{\mathbf{1}} \rightarrow \mathbf{k}_{\mathbf{3}}\right) \hat{\mathbf{P}}_{\mathbf{n}-\mathbf{1}}\left(\mathbf{n}-\mathbf{1}, \mathbf{k}_{\mathbf{1}}\right)\right)
\end{gathered}
$$

where $\hat{\mathbf{k}}_{\mathbf{n}}\left(\mathbf{n}, \mathbf{k}_{\mathbf{3}}\right)$ and $\hat{\mathbf{P}}_{\mathbf{n}}\left(\mathbf{n}, \mathbf{k}_{\mathbf{3}}\right)$ are quantities associated to the state $k_{3}$ as well as $\hat{\mathbf{c}}_{\mathbf{n}+\mathbf{1}}\left(\mathbf{k}_{\mathbf{3}}\right)$. The $\hat{\mathbf{P}}_{\mathbf{n}}(n, k)$ represents path inverse autocorrelation matrices and must be stored one per state for iterative updating. Notice that formally the per-survivor processing results in an extension of Viterbi algorithm. Associated to each survivor path we don't find any longer just a metric, but a metric 
and other parameters (the branch function coefficients vector $\hat{\mathbf{c}}_{\mathbf{n}+\mathbf{1}}(k)$ and, if RLS is used, the path inverse autocorrelation matrix $\left.\hat{\mathbf{P}}_{\mathbf{n}}(n, k)\right)$. This feature makes the per-survivor approach attractive from a conceptual point of view although a theoretical comparison (in terms of expected performance) with the delayed min-survivor approach is not easy to attain.

The LMS and RLS algorithms are used in the particular context of additive Gaussian- distributed noise, in which the squared output error is minimized. Therefore if the true transmitted L-uple $\mathbf{a}_{\mathbf{n}}$ is employed for coefficients updating at step $n$, since

$$
\mathbf{y}_{\mathbf{n}}=\mathbf{a}_{\mathbf{n}}^{\mathbf{T}} \mathbf{c}_{\mathbf{n}}+\mathbf{n}_{\mathbf{n}}
$$

where $\mathbf{n}_{\mathbf{n}}$ are AWGN samples, the corresponding adjustment term can directly estimate the true coefficients vector $\mathbf{c}_{\mathbf{n}}$. On the other hand when a generic L-uple $\alpha_{\mathbf{n}}$ is employed, then the corresponding received signal model results in

$$
\mathbf{y}_{\mathbf{n}}=\alpha_{\mathbf{n}}^{\mathbf{T}} \mathbf{c}_{\mathbf{n}}+\mathbf{m}_{\mathbf{n}}
$$

where the additive noise term

$$
\mathbf{m}_{\mathbf{n}}=\mathbf{n}_{\mathbf{n}}+\left(\mathbf{a}_{\mathbf{n}}-\alpha_{\mathbf{n}}\right)^{\mathbf{T}} \mathbf{c}_{\mathbf{n}}
$$

is no longer Gaussian-distributed, therefore the over mentioned algorithms can even not correctly determine the adjustment term at step $\mathrm{n}$. Let us introduce the diagonal matrix $\mathbf{E}_{\mathbf{n}}\left(\alpha_{\mathbf{n}}\right)=\operatorname{diag} \mathbf{a}_{\mathbf{n}} \operatorname{diag} \alpha_{\mathbf{n}}$ whose generic element is: $e_{(i, j)}=0$ for $i \neq j(i, j=0, . ., L-1)$;

$e_{(i, i)}=+1$ if $\alpha_{n}(i)$ is "right" when compared to the corresponding true transmitted symbol $\mathbf{a}_{\mathbf{n}}(i)$ and

$e_{(i, i)}=-1$ if it is "wrong" $(i=0, . ., L-1)$.

It is easy to see that the signal model, if the $\alpha_{n}$ L-uple and additive noise AWGN are transmitted, can be written as

$$
\mathbf{y}_{\mathbf{n}}=\alpha_{\mathbf{n}}^{\mathbf{T}} \mathbf{E}\left(\alpha_{\mathbf{n}}\right) \mathbf{c}_{\mathbf{n}}+\mathbf{n}_{\mathbf{n}}
$$

The adjustment term moves the estimation at step $n$ towards $\mathbf{E}_{\mathbf{n}}\left(\alpha_{\mathbf{n}}\right) \mathbf{c}_{\mathbf{n}}$ which disagrees with $\mathbf{c}_{\mathbf{n}}$ having opposite component values in correspondence to wrong components in $\alpha_{\mathbf{n}}$. Now, when the per-survivor estimation is carried out at step $n$, since all the L-uple $\alpha_{\mathbf{n}}$ associated to the $2^{L-1}$ survivors differ from one other, it follows that at least one of the updating terms associated to each survivor as well is correct and the estimation moves in the right direction. This statement does not imply that the per-survivor estimation paths are globally diverging. In fact at each step the global estimation history is similar for all the survivor paths since all of them are quickly converging backwards in the past [5]. Only in the steps preceding step $n$ the estimation paths tend to diverge. Particularly, the more errors appear inside a path in the most recent bits (particularly in 
the L-1 state bits), the more the final estimated coefficients associated to it may differ from the right ones. This is not, in general, a favorable condition for a correct demodulation, since some wrong survivor path could even worsen the bad estimation associated to them: e.g. the path corresponding to $-\left\{a_{n}\right\}$ sequence, if surviving, could lead quite quickly the coefficients estimation to its equilibrium point $-\mathbf{c}_{\mathbf{n}}$ and survive further. Besides, statistics estimated through computer simulations (see next section) have shown that the survivor metrics (shifted at each step by their minimum value, in such a way that the smallest is always zero) exhibit smaller average and standard deviation values when the estimation technique is per-survivor instead then min-survivor. This condition may indicate a smaller ability in emphasizing different likelihood paths by the per-survivor technique. The MLSE principle simply states that the correct coefficients should be employed in the computation of all the branch metrics. Furthermore, a hypothesis of continuity in the receiver error probability dependence from the coefficients error is certainly reasonable to be assumed. Again, this is not a reason in favor of per-survivor processing.

Finally, the computational complexity comparison for the two considered estimation techniques indicates that a significant amount of computation can be avoided using the min-survivor technique. The reason lies substantially in the fact that the min-survivor processing carries out one estimation process instead of $2^{L-1}$ (one per state). Particularly, the basic cost of a single vector of coefficient updating depends on the algorithm used (LMS or RLS) and can be expressed as the sum $N_{s} c_{s}+N_{m} c_{m}+N_{c} c_{c}$ where $N_{s}, N_{m}, N_{c}$ are the real sums, real multiplications and memory cells required and $c_{s}, c_{m}, c_{c}$ are their unitary costs. (See Table 4 ). Table 4 lists the values $N_{s}, N_{m}, N_{c}$ as a function of $\mathrm{L}$. The computational complexity for the error $e_{n}$ is not included in Table 4 as this value is available by the Viterbi processor. The cost of min-

\begin{tabular}{||l|l|l|l||}
\hline & $N_{s}$ & $N_{m}$ & $N_{c}$ \\
\hline \hline LMS & $2 \mathrm{~L}$ & 2 & $2 \mathrm{~L}$ \\
\hline RLS & $2 L^{2}+2 L$ & $2 L^{2}+3 L$ & $L^{2}+4 L$ \\
\hline \hline
\end{tabular}

Table 4 Numbers $N_{s}, N_{m}, N_{c}$ of real sums, multiplications, memory cells required by one vector of coefficients updating

survivor processing is then determined by three additional quantities: the first, $c_{1}=\left(2^{L-1}-1\right) c_{s}+c_{c}$ is due to the minimum survivor search problem solution; the second, $c_{2}$ depends on the particular memory allocation of the survivor path (in any case linear with respect to L parameter) and is due to the presence of delay $d$ in $38-41$, which requires to look backwards in the minimum survivor 
path to find the bit vector $\hat{a}_{n-d}(n)$; the third, $c_{3}=L c_{s}+c_{c}$ is required to evaluate the output error $e_{n-d}\left(\hat{a}_{n-d}(n), \hat{c}_{n}\right)$. As a result, the estimation cost for min-survivor is per step

$$
c_{\text {minsurv }}=\left(N_{s} c_{s}+N_{m} c_{m}+N_{c} c_{c}\right)+c_{1}+c_{2}+c_{3}
$$

On the other hand the per-survivor computational complexity is $2^{L-1}$ times the basic cost, that is per step:

$$
c_{\text {persurv }}=2^{L-1}\left(N_{s} c_{s}+N_{m} c_{m}+N_{c} c_{c}\right)
$$

Considering the values shown in Table 4 for $N_{s}, N_{m}, N_{c}$, the persurvivor, minsurvivor computational complexities ratio asintotically grows with a power of L (which is 1 for LMS and 2 for RLS), in such a way that:

$$
\lim _{L \rightarrow \infty} \frac{1}{L} \frac{c_{\text {persurv }}(L)}{c_{\text {minsurv }}(L)}=\frac{2 c_{c}}{c_{s}}+2
$$

for LMS, and:

$$
\lim _{L \rightarrow \infty} \frac{1}{L^{2}} \frac{c_{p e r s u r v}(L)}{c_{\text {minsurv }}(L)}=\frac{2 c_{m}}{c_{s}}+\frac{c_{c}}{c_{s}}+2
$$

for RLS.

\section{Simulated performance}

\subsection{Simulated system}

The performance of the different adaptive MLSE receivers have been experimentally evaluated according to the standard of the new high capacity pan European digital mobile radio system, the ETSI/GSM. This system [14],[16],[19] employees a TDMA with 8 channels per carrier. All the carriers are located in the $900 \mathrm{M} \mathrm{Hz}$ frequency band and are spaced by a $200 \mathrm{kHz}$ bandwidth. In the case of user data packet (normal burst), the time slot basic structure is depicted in fig. 8. The bits are usually referred to in terms of their logical 0 and 1 values, while the corresponding physical ones are +1 and -1 . The information bits are split into two groups of 58 bits by a 26 -bit long preamble sequence that enables the synchronization and the estimate of the channel response. The midamble sequence $m_{n} \quad n=1, . ., 26$ (physical values) is characterized by a particular autocorrelation property, that is

$$
\frac{1}{16} \sum_{k=5}^{20} m_{k} m_{n+k}=\delta_{n} \quad n=-4, \ldots, 0, . ., 4
$$




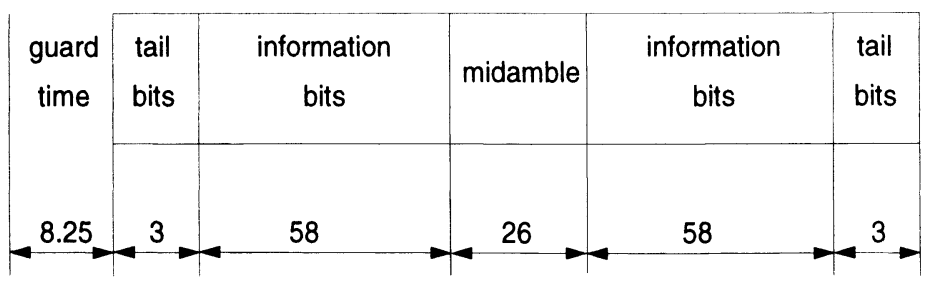

time (bit period, $\mathrm{T}$ )

Fig. 8 GSM normal burst structure

This autocorrelation [26] property makes optimal for training estimation of system with no more of $\mathrm{L}=5$ coefficients. The bit period evaluates $T=3.69 \mu \mathrm{s}$, hence the bit rate is $R=270.833 \mathrm{kbit} / \mathrm{s}$. The adopted modulation scheme is the GMSK signaling with normalized 3 - $\mathrm{dB}$ bandwidth $B T=0.3$ which is characterized by modulation index $h=1 / 2$, a smooth shaping pulse, narrow band, and belongs to the class of CPM described in Section 2. Before entering the GMSK phase modulator, the binary sequence is differentially encoded as indicated in Section 2, so that the linear model 6,7 can be used for transmitted signal. In order to assess performance and compare different MLSE receivers various propagation models have been considered:

- AWGN channel;

- AWGN channel with Doppler effect $(175 \mathrm{~Hz}$ carrier frequency constant shift corresponding to vehicle radial velocity $210 \mathrm{~km} / \mathrm{h}$ );

- the fading multipath channels indicated as TU (Typical Urban), HT (Hilly Terrain) and RA (Rural Area) in GSM Recommendations are simulated by the 6 specified taps with mobile speed of respectively $50 \mathrm{~km} / \mathrm{h}, 100$ $\mathrm{km} / \mathrm{h}$ and $300 \mathrm{~km} / \mathrm{h}$. 
The employed transmitter and receiver bandpass filters are linear phase ones. The transmitter filter has been chosen with an enough wide band not to modify the signal shape. According to the equivalent baseband representation, the receiver filter features are depicted in 9,10, 11 illustrate respectively the shape of linearized baseband transmitted and filtered pulse. Finally, the additive
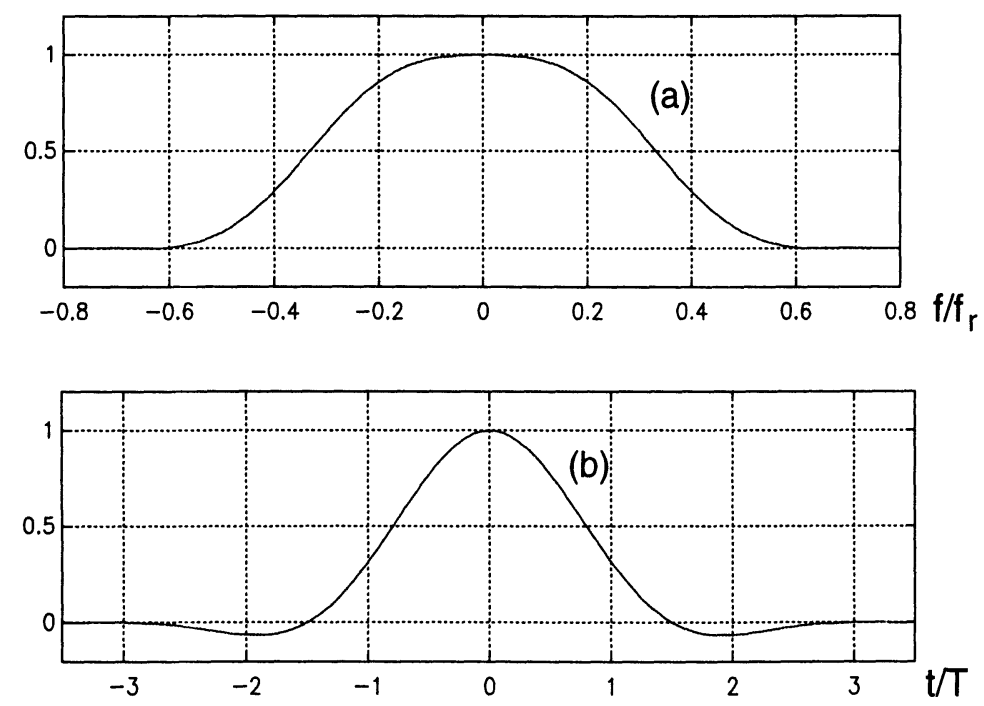

Fig. 9 Receiver filter: (a) frequency domain amplitude response; (b) time domain impulse response

noise introduced by the channel simulator was created as white. Signal to noise ratios values of $3,5,8 \mathrm{~dB}$ were simulated in the AWGN and AWGN with Doppler channels, while $8,16,24 \mathrm{~dB}$ were chosen for simulation in TU, HT and $\mathrm{RA}$. The receiver sampling frequency has been chosen equal to bit rate $(\mathrm{C}=1)$, and the parameter $\mathrm{L}$ has been regularly set to a value of 5 for the trials, in such a way that the simulated demodulator were always a Viterbi $2^{L-1}=16$ states one. The values for the channel coefficients $c(i), i=0, . ., 4$ resulting from the modulation scheme, the filters and the synchronization constants, are listed in Table 5. The performance comparison among the 'non adaptive' receiver,the LMS/RLS min-survivor receiver and the LMS/RLS per-survivor receiver is evaluated in terms of the bit error rate (BER) versus $E_{b} / N_{0}$. In the 'non adaptive' receiver the known training sequence of each burst (midamble) is used to estimate the channel response during the actual burst. This training channel estimate is held fixed in the computing of the Viterbi metric for the entire burst duration and is updated only the next burst. The non adaptive term is used 

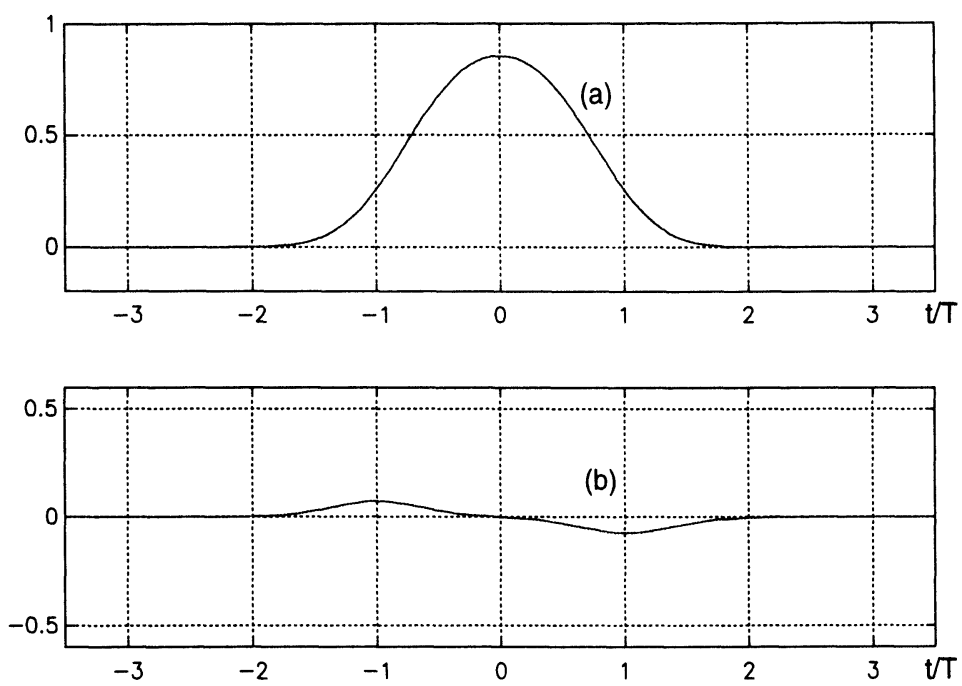

Fig. 10 Transmitted pulse shape: (a) real part; (b) imaginary part

\begin{tabular}{||l||l|l||}
\hline \hline coeff. & real part & imag.part \\
\hline \hline$c(0)$ & 0.02 & 0.37 \\
\hline$c(1)$ & 0.04 & -0.71 \\
\hline$c(2)$ & -0.02 & 0.37 \\
\hline$c(3)$ & 0 & -0.03 \\
\hline$c(4)$ & 0 & 0 \\
\hline \hline
\end{tabular}

Table 5 c(i) coefficients (sampled received pulse) for GMSK BT=0.3 modulation and adopted receiver filter

in this section to indicate a periodic update of the channel parameters (at each burst). It differs from the adaptive receivers (LMS/RLS min-survivor receiver or per-survivor receiver) which use this training channel estimate only for start up purposes and then update continuously the channel estimate within the burst. The detection of the transmitted data sequence is performed by the Viterbi algorithm at the end of each burst for the non-adaptive and adaptive 

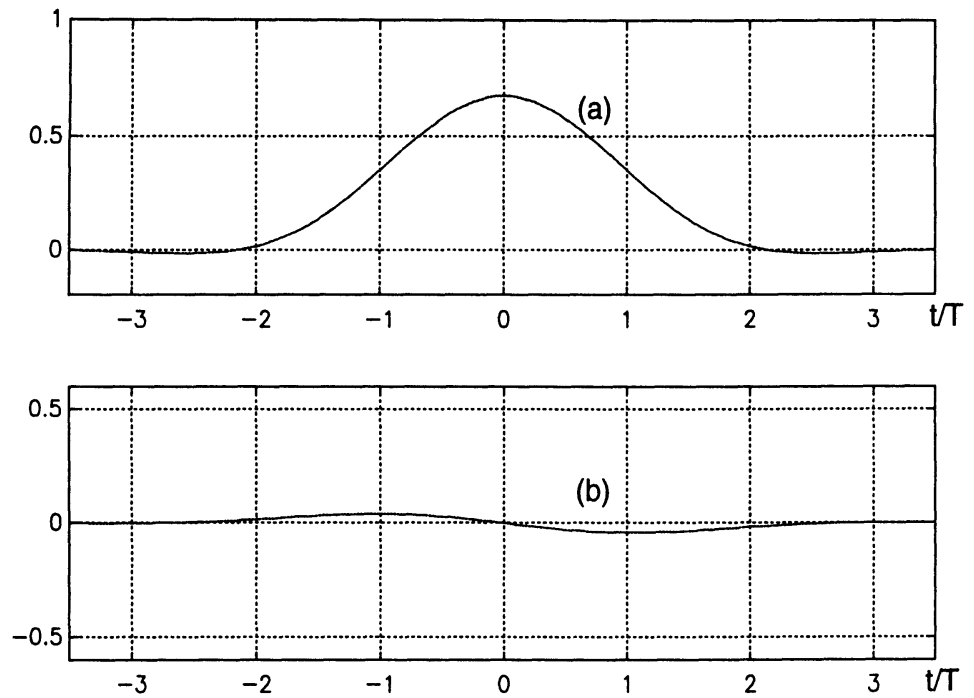

Fig. 11 Received pulse shape:(a)real part, (b) imaginary part

receivers. Let

$$
y_{n}=\sum_{i=0}^{4} m_{n-i} c(i)+n_{n}
$$

the received signal in correspondence of midamble, where $n_{n}$ is the AWGN term (variance $\sigma^{2}$ ).

The channel training estimation is defined as the signal-midamble cross correlation

$$
\hat{c}_{\text {training }}(i)=\frac{1}{16} \sum_{n=5}^{20} y_{n+i} m_{n} \quad i=0, \ldots, 4
$$

Substituting 54 into the last one, in the hypothesis $c(i) \neq 0$ only for $0 \leq i \leq 4$, yields

$$
\hat{c}_{\text {training }}(i)=c(i)+\epsilon(i) \quad i=0, \ldots, 4
$$

where

$$
\epsilon(i)=\frac{1}{16} \sum_{n=5}^{20} m_{n-i} n_{n} \quad i=0, \ldots, 4
$$

are Gaussian distributed errors with variance $\sigma^{2} / 16$. The performance of the different receivers has been assessed by demodulating the same signal and noise realization for each assigned channel type and $E_{b} / N_{0}$. The statistics extension 
is determined by the number of transmitted burst simulated, which has been fixed on 1000 bursts. The results presented refer to optimized values of the estimation algorithms parameters. The optimization was carried out experimentally repeating the demodulation trials for different parameters values. For LMS algorithm, the optimal value of the step size parameter $\mu$ has been found to range from $1 / 80$ to $1 / 20$ in any case. For RLS algorithm in the AWGN channel case, according with theory, the optimality for the forgetting factor $w$ is exhibited for $\mathbf{w}$ close to 1 i.e. 0.9999 ; in the other cases, optimality is exhibited in the range $0.96 \div 0.99$. When min-survivor estimation technique was employed, the $d$ delay parameters has been optimized as well. The values chosen were $d=1 T$ for AWGN, AWGN and Doppler effect, RA channel and $d=2 T$ for TU, HT channels.

Finally, the behavior of the used adaptive algorithms (RLS and LMS) within the Viterbi algorithm is compared in the case of a continuous sequence transmission with the GMSK modulation.

\subsection{Results}

\subsubsection{AWGN channel}

The BER simulated performance comparisons between nonadaptive, persurvivor, minsurvivor, reference receivers are shown in fig.12, fig. 13 respectively when LMS and RLS algorithm are employed. The reference curves are associated to the receiver that uses permanently the correct likelihood parameters (see Table 5). These results show that all the LMS/RLS minsurvivor receiver and LMS/RLS persurvivor receiver exhibit very similar performance. The adaptive techniques allow to improve the performance of non-adaptive receivers, although they do not attain the optimal reference receiver one.

This behavior points out that:

1. The noise on received signal determines sensible degradation in training (midamble) estimation and, hence, BER degradation of the nonadaptive receiver with respect to the reference receiver. The adaptive parameters estimation techniques, by means of parameters improvement, recover only a small part of the non-adaptive receiver errors.

2. The residual BER gap between reference and adaptive receivers is due to the finite time-delay in the convergence process of tracking. 


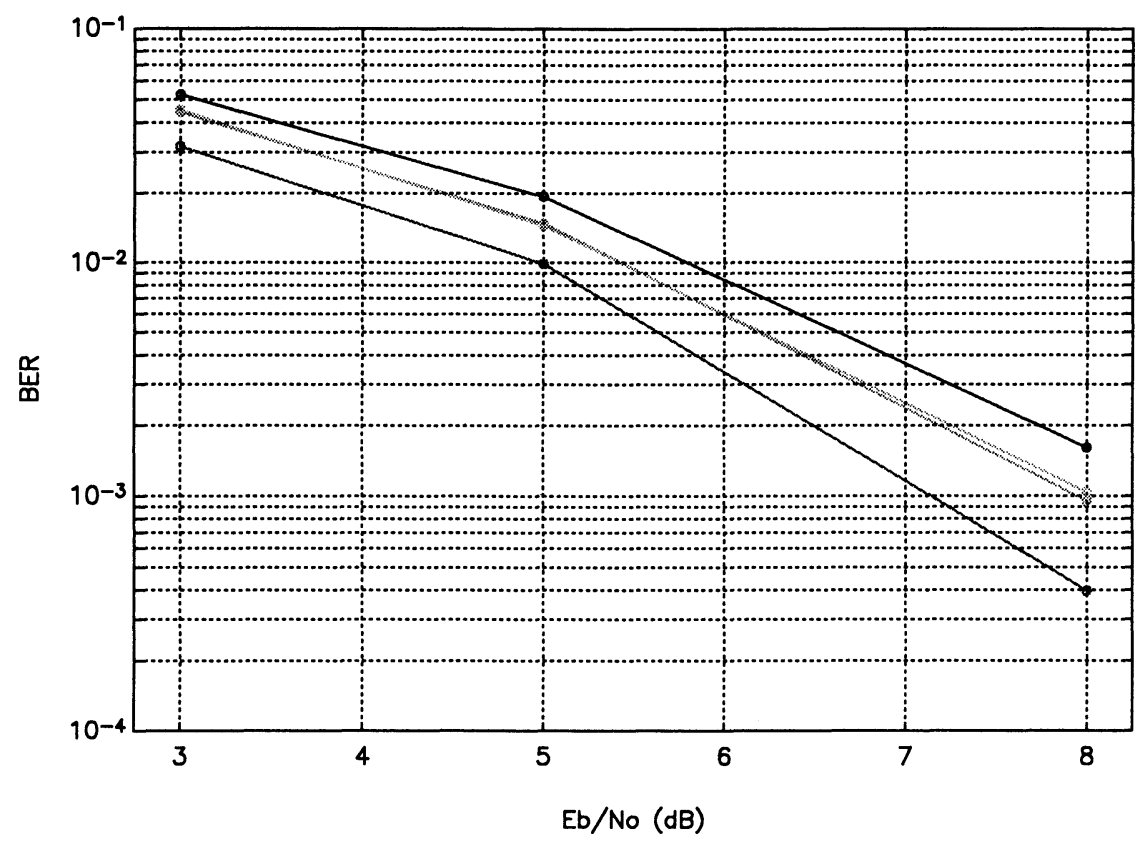

Fig. 12 AWGN channel, Euclidean MLSE performance (BER) : (a) non adaptive; (b) LMS min-survivor (c)LMS per-survivor; (d) reference receiver. 


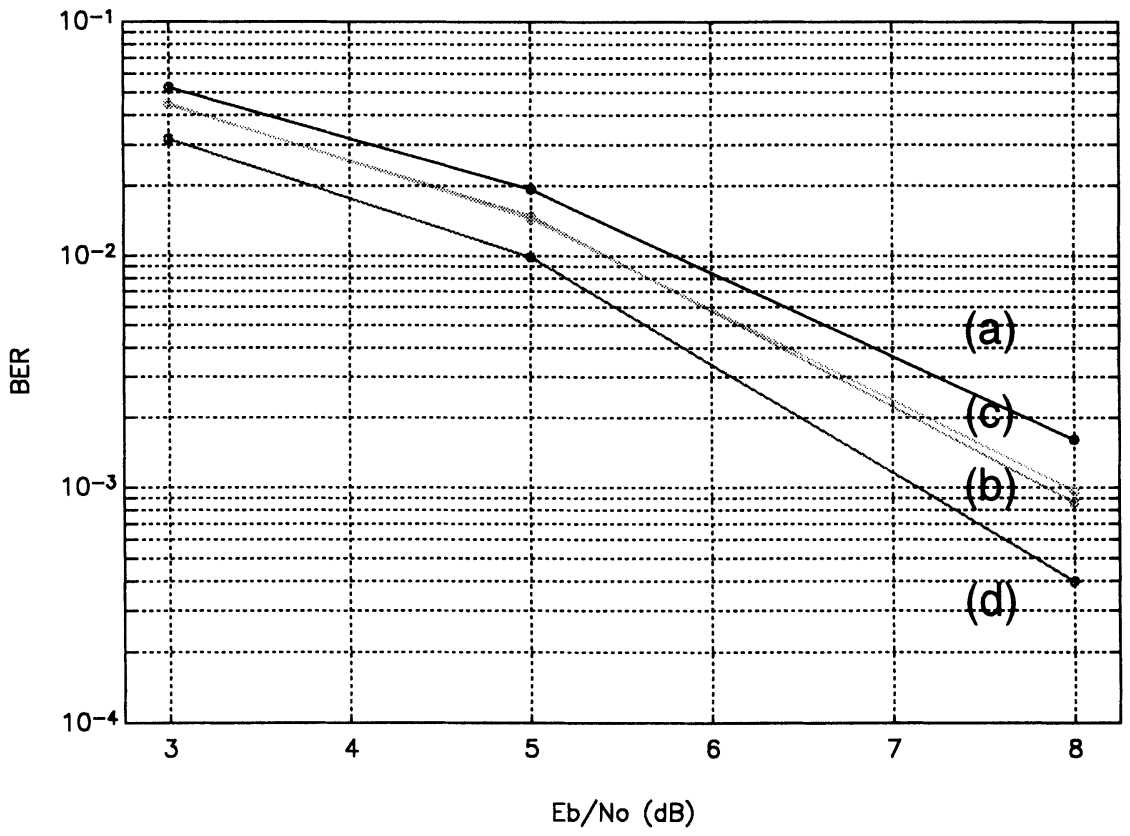

Fig. 13 AWGN channel- Euclidean MLSE BER : (a) non adaptive; (b) RLS min-survivor (c)RLS per-survivor; (d) reference receiver. 

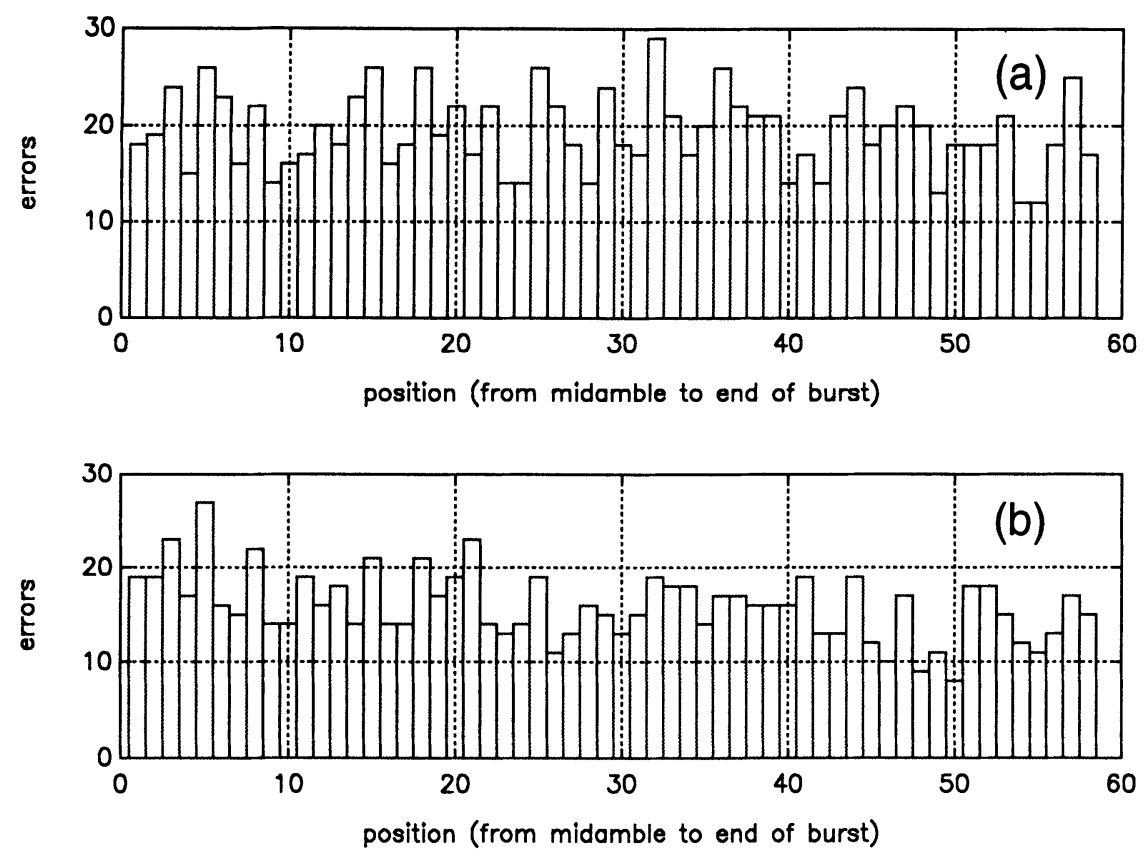

Fig. 14 AWGN channel- $E_{b} / N_{0}=5 d B$ histogram of errors ( 1 is the midamble side and 58 is the end of burst), statistic on 1000 bursts: (a) non adaptive receiver; (b) LMS min-survivor $\mu=1 / 20$.

As shown in fig.14, the adaptive receiver exhibits a statistic of errors similar to the non adaptive one. Fig. 15 depicts the comparison of average mean and standard deviation (shifted at each step by their minimum value, in such a way that the smallest is always zero) of the survivor metrics between per-survivor and min-survivor adaptation techniques: as seen, the min-survivor receiver exhibits greater values. Notwithstanding this, no preference can be given from observation on simulated BER performance.

\subsubsection{AWGN Channel with Doppler effect}

These demodulation simulations refer to an impairment introduced by multiplying the transmitted signal (its complex envelope) by the exponential $e^{j 2 \pi f_{d} t}$, with $f_{d}=175 \mathrm{~Hz}$ (corresponding to $210 \mathrm{~km} / \mathrm{h}$ vehicle speed, which is a $16^{\circ}$ phase shift in half burst), in order to describe the progressive rotation of received pulse due to the presence of Doppler effect. The results are shown in 


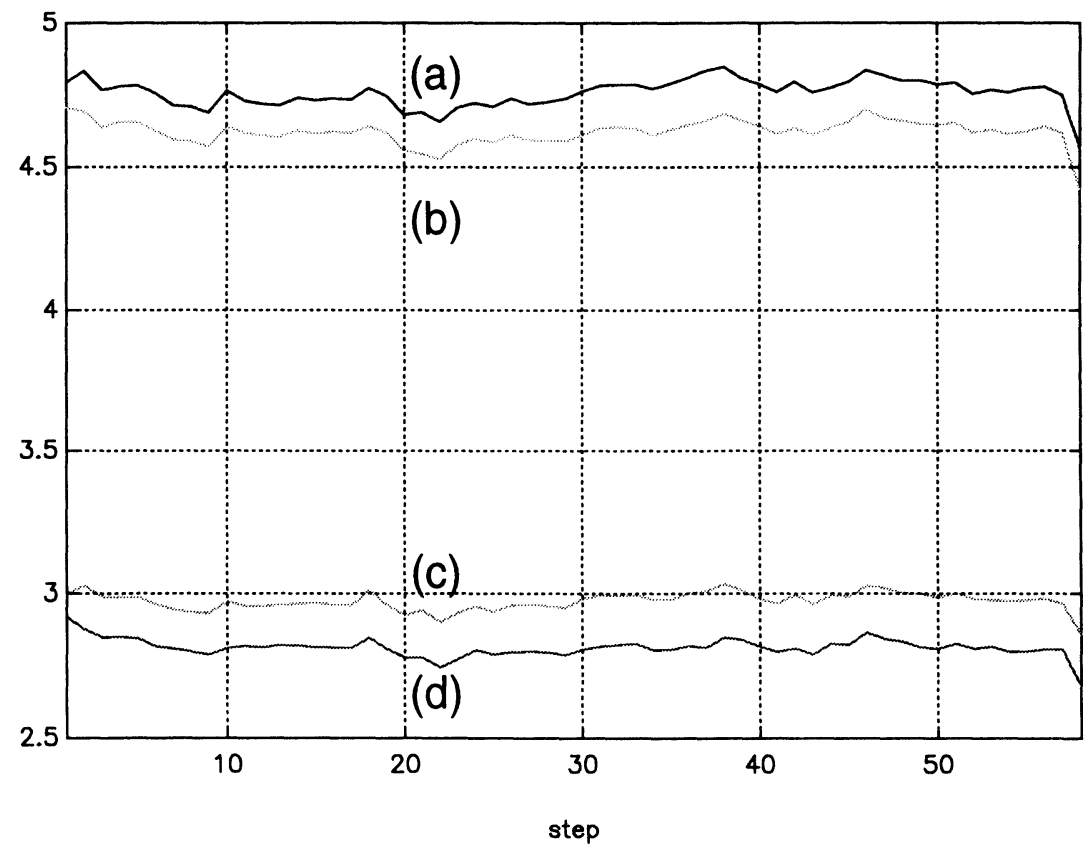

Fig. 15 AWGN channel- $E_{b} / N_{0}=5 d B$, LMS $\mu=1 / 20$ average mean and standard deviation of survivor metrics on 1000 bursts : (a) min-surv mean; (b) per-surv mean; (c)min-surv std. dev; (d) per-surv std. dev. 
fig.16 17. The reference curve is the same as in fig. 12,13 without Doppler effects. As seen, the non adaptive receiver performance degrades with respect to AWGN channel case. The adaptive techniques allow an effective tracking of parameters. A $2 \mathrm{~dB}$ improvement is attained with respect to non-adaptive receiver at $B E R=210^{-3}$. This result is confirmed by the histograms depicted in fig.18: while the adaptive receivers keep constant the BER ( the tracking processing already begins in the midamble sequence), the non-adaptive demodulation errors grow from midamble to the end of burst.

\subsubsection{GSM RA channel}

In this environment, the fading and Doppler effects due to the high vehicle speed (simulated speed: $300 \mathrm{~km} / \mathrm{h}$ ), contribute to the degradation of the transmitted signal. The multipath effect does not change the channel response length significantly since the maximum simulated delayed path arrives to the receiver antenna $1 / 7 \mathrm{~T}$ later than the direct path. However, the multipath degrades the BER of the receiver. The simulation results are presented in fig.19, fig. 20 . A performance improvement of $5 \mathrm{~dB}$ at BER equal to $10^{-2}$ is exhibited for adaptive receivers versus non-adaptive ones.

\subsubsection{GSM TU Channel}

This environment is characterized by moderated Doppler effect (simulated vehicle speed: $50 \mathrm{~km} / \mathrm{h}$ ), and multipath effect (1.3T maximum simulated delay) due to presence of significant reflectors like large buildings walls. Fig. 2122 depict the performance comparison respectively for LMS and RLS adaptive receiver with non-adaptive receiver. The improvement for adaptive receivers reaches 3 - $\mathrm{dB}$ at $\mathrm{BER}$ equal to $4 \times 10^{-4}$. One more time, no relevant difference are observed between LMS/RLS algorithms and minsurvivor persurvivor techniques.

\subsubsection{GSM HT channel}

In this model the vehicle speed is equal to $100 \mathrm{~km} / \mathrm{h}$. Due to large time delays determined in some paths by the signal reflection on the mountains surfaces according to the GSM specifications, relevant amount of energy arrives to the receiver antenna even $8 \mathrm{~T}$ later than the direct path. This energy results in additive noise since the length of the adopted Viterbi memory $(L=5)$ is not sufficient to recover such a large multipath effect. Moreover using a Viterbi 


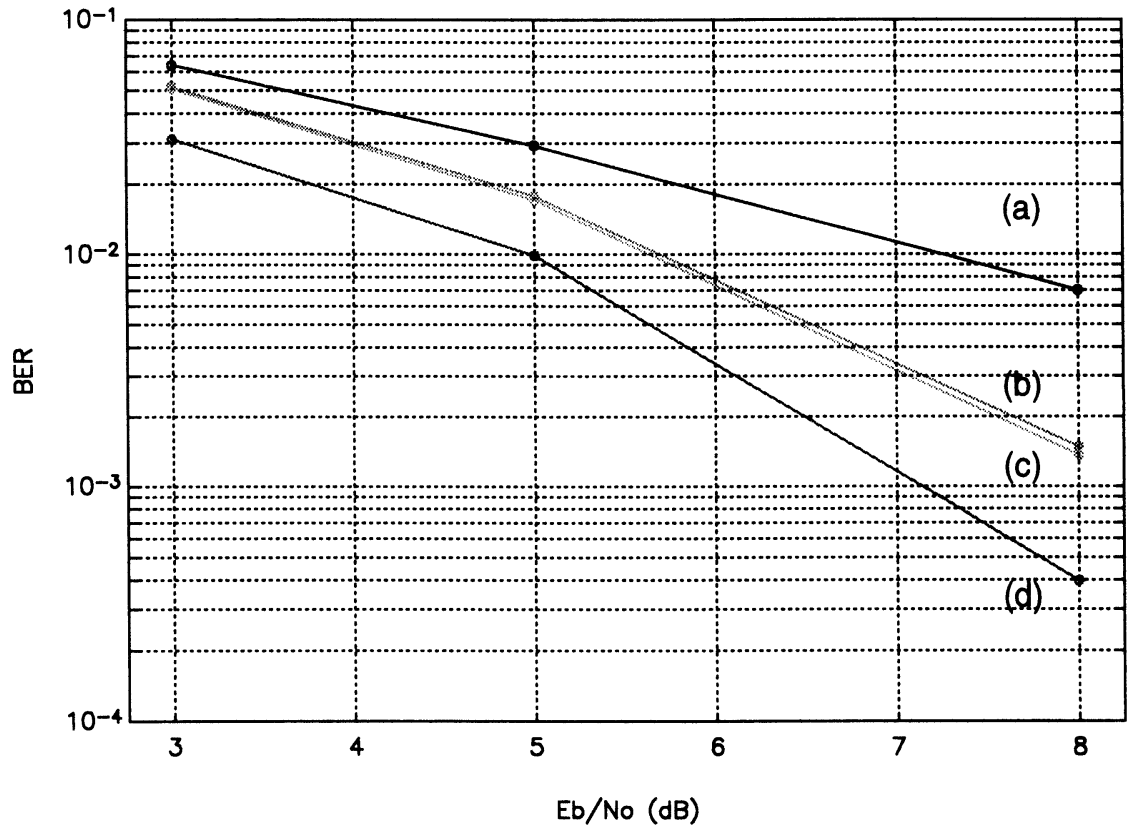

Fig. 16 AWGN channel with Doppler effect-Euclidean MLSE BER: (a) non adaptive; (b) LMS min-survivor; (c) LMS per-survivor; (d) reference receiver. 


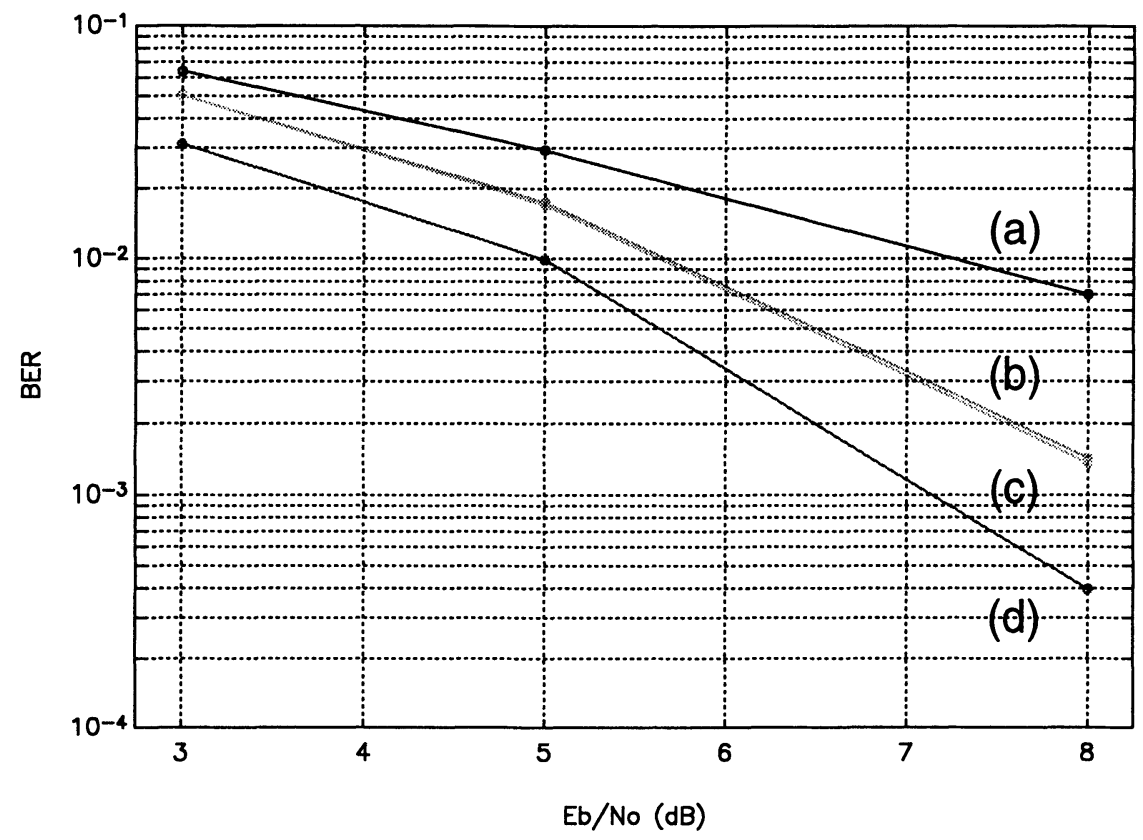

Fig. 17 AWGN channel with Doppler effect-Euclidean MLSE BER: (a)non adaptive; (b) RLS min-survivor; (c) RLS per-survivor; (d) reference receiver. 

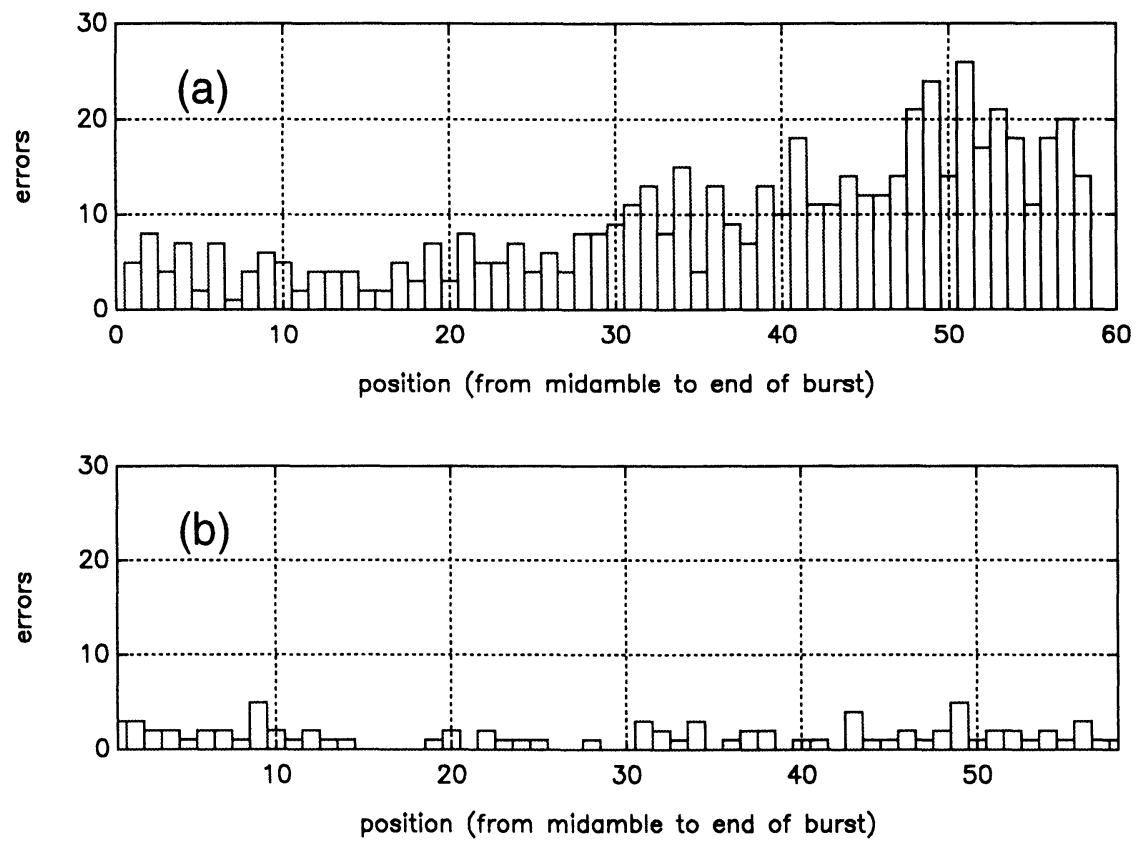

Fig. 18 AWGN channel with Doppler effect- $E_{b} / N_{0}=5 d B$ histogram of errors ( 1 is the midamble side and 58 is the end of burst), statistic on 1000 bursts: (a) non adaptive receiver; (b) RLS min-surv. $w=0.96$. 


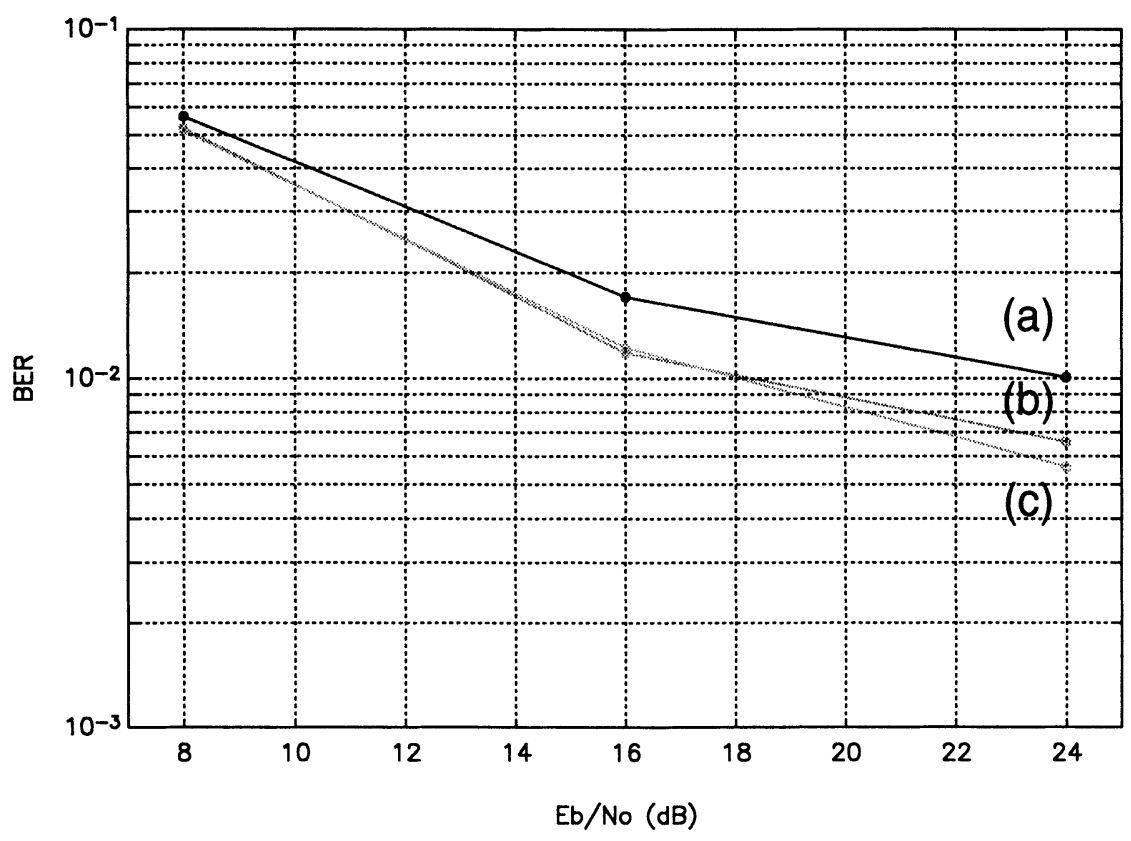

Fig. 19 GSM RA channel- Euclidean MLSE BER: (a) non adaptive receiver; (b) LMS min-survivor; (c) LMS per-survivor. 


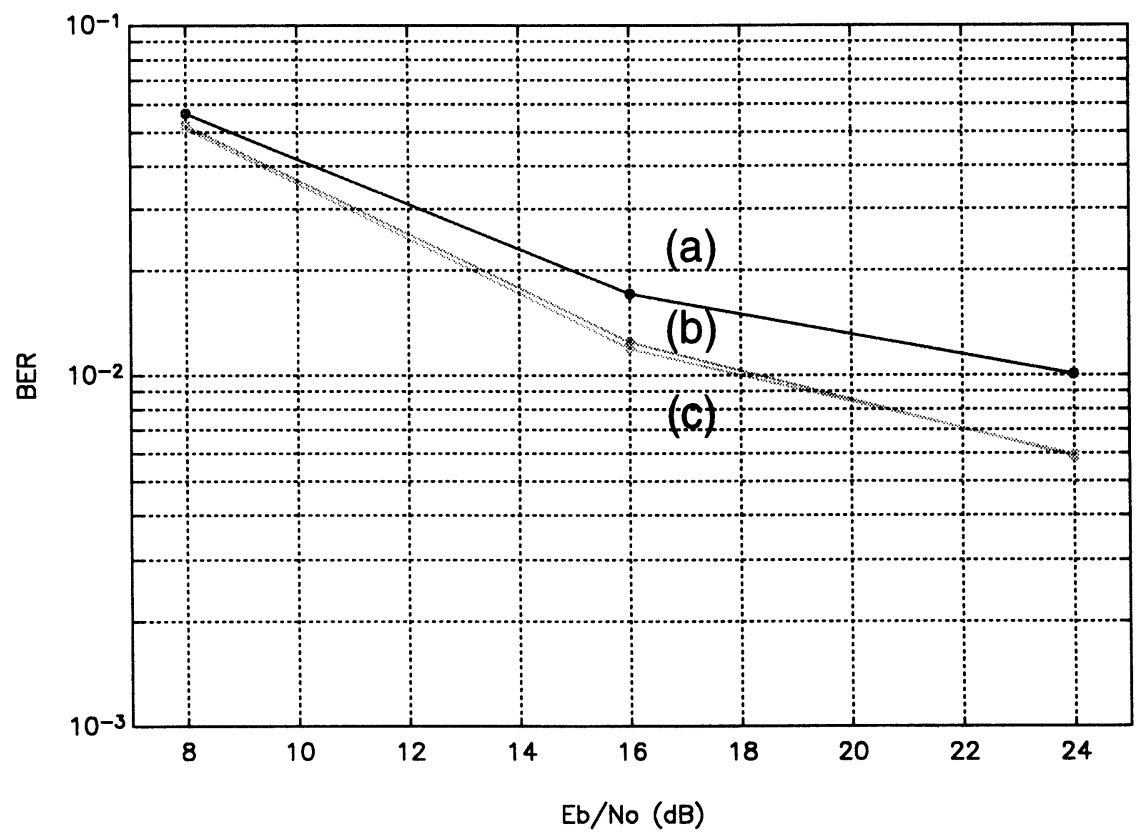

Fig. 20 GSM RA channel- Euclidean BER: (a) non adaptive receiver; (b) RLS min-survivor; (c) RLS per-survivor. 


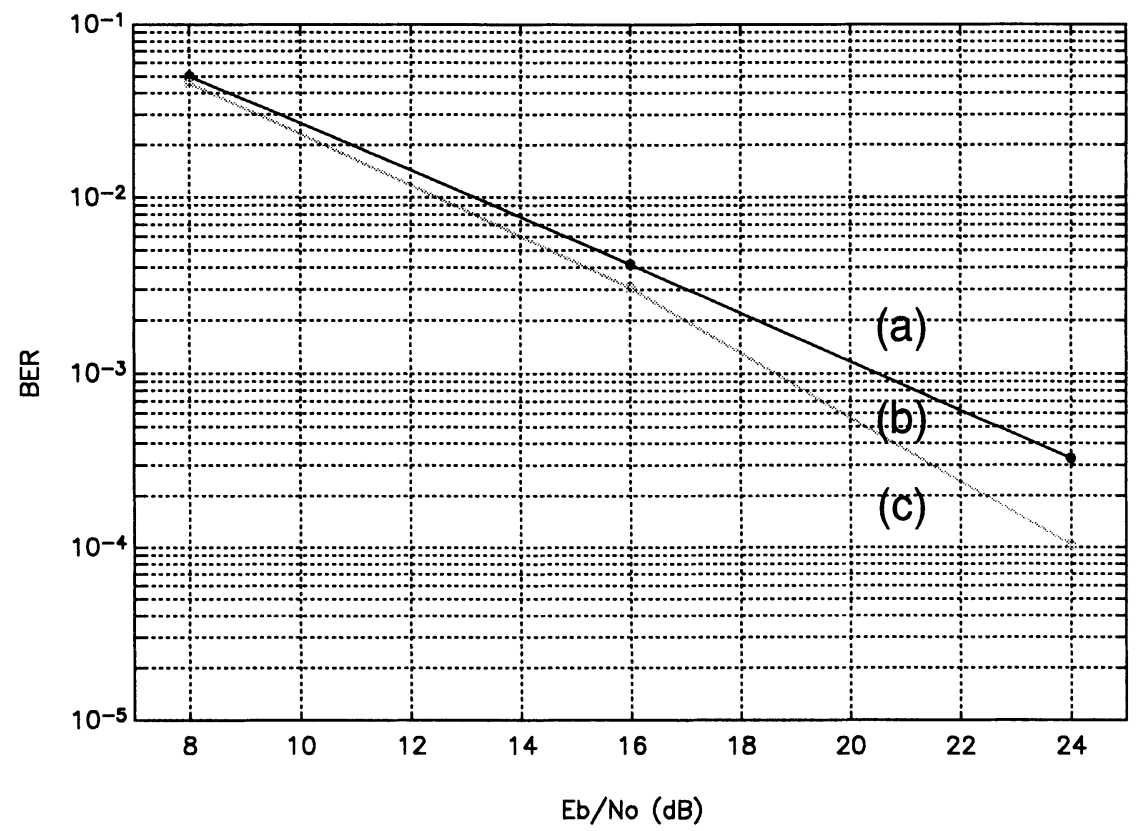

Fig. 21 GSM TU channel- Euclidean MLSE BER: (a) non adaptive receiver; (b) LMS min-survivor; (c) LMS per-survivor. 


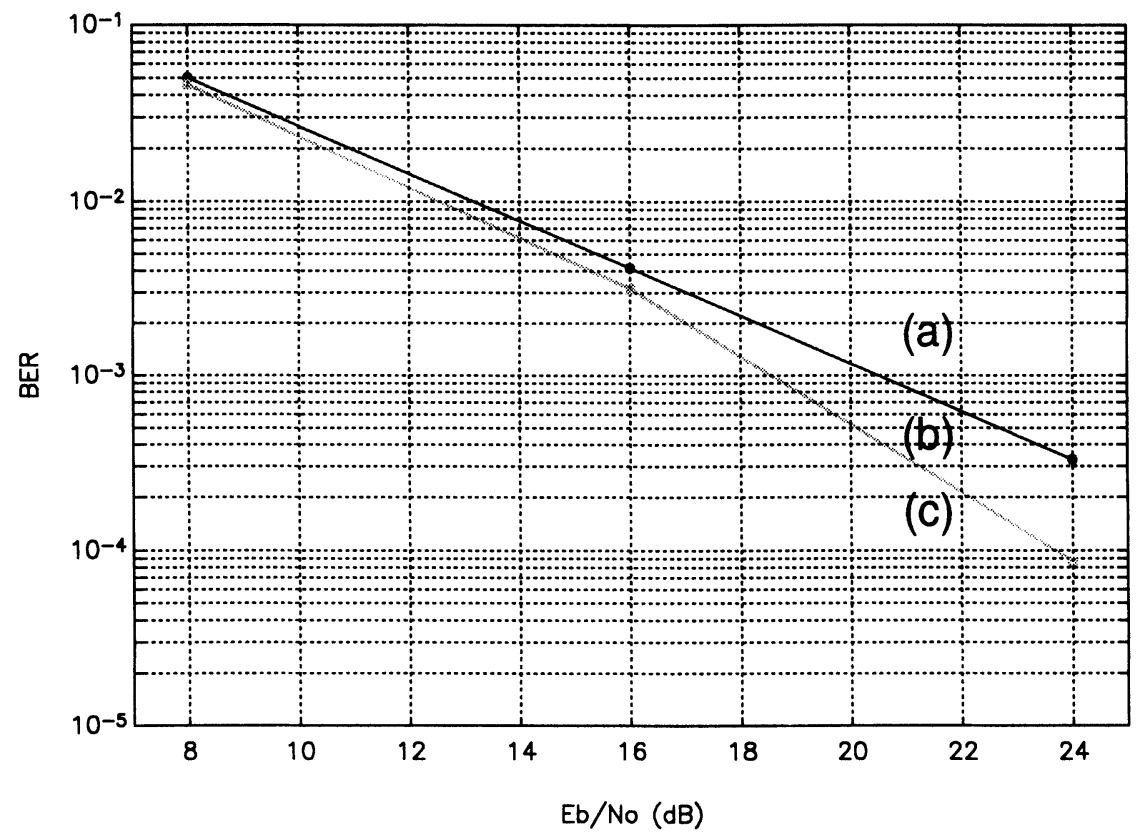

Fig. 22 GSM TU channel- Euclidean MLSE BER: (a) non adaptive receiver; (b) RLS min-survivor; (c) RLS per-survivor. 
receiver with memory $L$ equal to 5 no improvement is obtained with adaptive techniques as shown in fig. 23,24 .

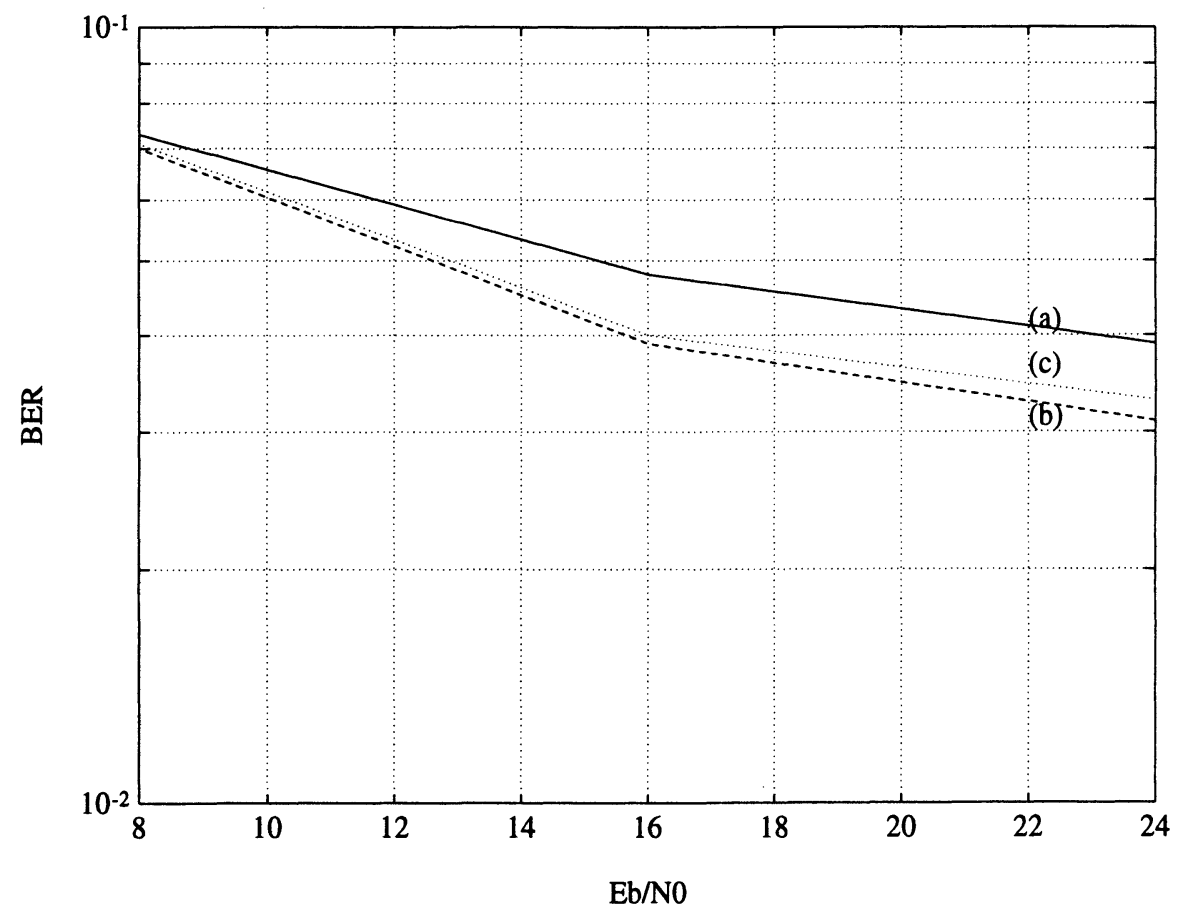

Fig. 23 GSM HT channel- Euclidean MLSE BER: (a) non adaptive receiver; (b) LMS min-survivor; (c) LMS per-survivor.

\subsection{Continuous modulations}

A continuous GMSK modulation has been simulated in order to compare the behavior of the different adaptive algorithms within the MLSE receiver. Particularly some meaningful adaptation learning curves have been found and reported in order to further distinguish the features of the different methods presented. A learning curve shows the maximum coefficients estimation error

$$
\max _{i=0, \ldots, 4}\left\{\left|c_{n}(i)-\hat{c}_{n}(i)\right|\right\}
$$




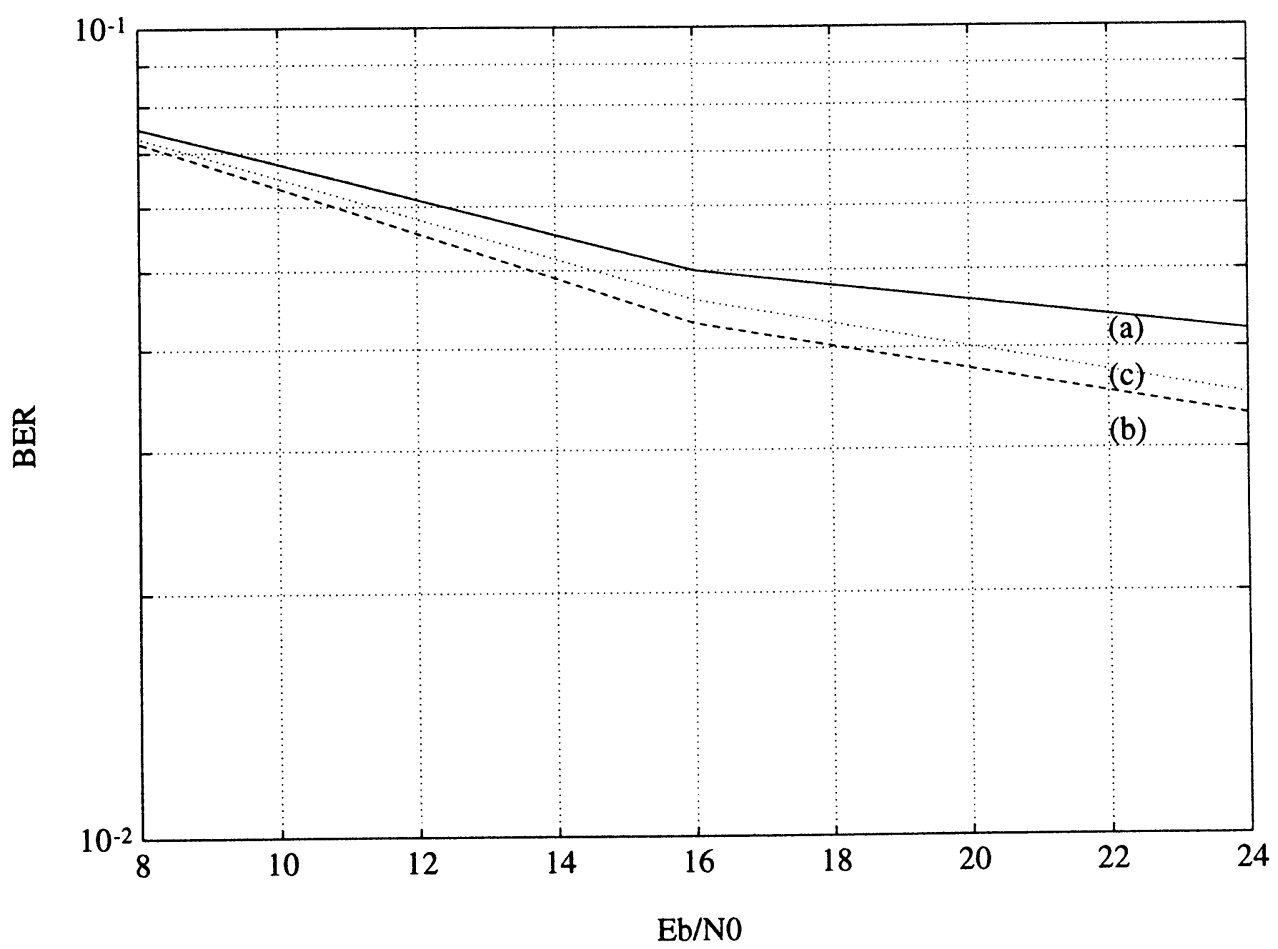

Fig. 24 GSM HT channel- Euclidean MLOSE BER: (a) non adaptive receiver; (b) RLS min-survivor; (c) RLS per-survivor.

versus the number of iterations $\mathrm{n}$ from parameters start-up. The continuous information data stream is preceded by a preamble transmission employed for (training) parameters start-up.

\subsubsection{AWGN channel}

Some learning curves for min-survivor processing are depicted in fig.25. The learning of the LMS algorithm proceeds as fast as the RLS learning while $\mu=1 / 20$.

On the other hand, for LMS to attain a residual mean estimation error as small as RLS, it is necessary to choose $\mu=1 / 320$. Instead, the RLS algorithm (with $\mathrm{w}=0.9999$ ) is optimal in terms of both speed of response and residual error. Notwithstanding this, in the small interval of steps 1-58 (a GSM information 
bits group) the LMS and RLS algorithms cannot show their different global performance if LMS is optimized with respect to speed of response $(\mu=1 / 20)$. However, the time constants of the estimation convergence process seems to evaluate about 1000 steps, which is more than the 58 steps duration of a GSM information bits group. Hence not the LMS nor the RLS algorithms can determine the optimal receiver demodulation performance if the GSM burst format is employed. Also the adaptive demodulation in the continuous modulation context has been simulated. The algorithms parameters were optimized with respect to mean residual error. The BER obtained on a 58000 bit sequence has been found to be equal to the reference receiver performance. Finally, fig. 26 depicts the comparison between min-survivor and per-survivor LMS and RLS optimal learning curves. The per-survivor learning curves are referred to the estimation path associated to the ML sequence. The average value of the represented curves evaluates $1.1 \times 10^{-3}$ for LMS $\mu=1 / 320$ and $1.6 \times 10^{-3}$ for RLS $\mathrm{w}=0.9999$. These results show that similar learning performance are exhibited by min-survivor and per-survivor estimation techniques.

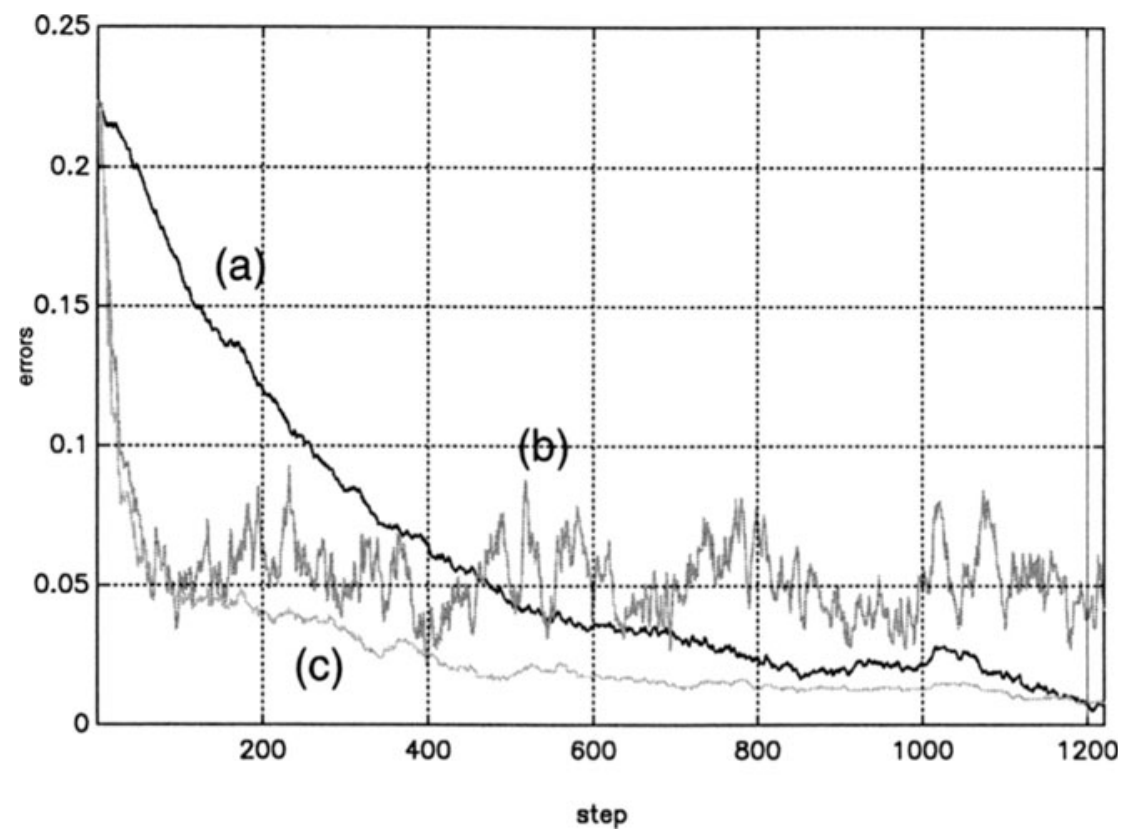

Fig. 25 Continuous transmission AWGN channel- $E_{b} / N_{0}=8 d B$ min survivor learning curves: (a)LMS $\mu 1 / 320$; (b) LMS $\mu=1 / 20$; (c) RLS w $=0.9999$ 


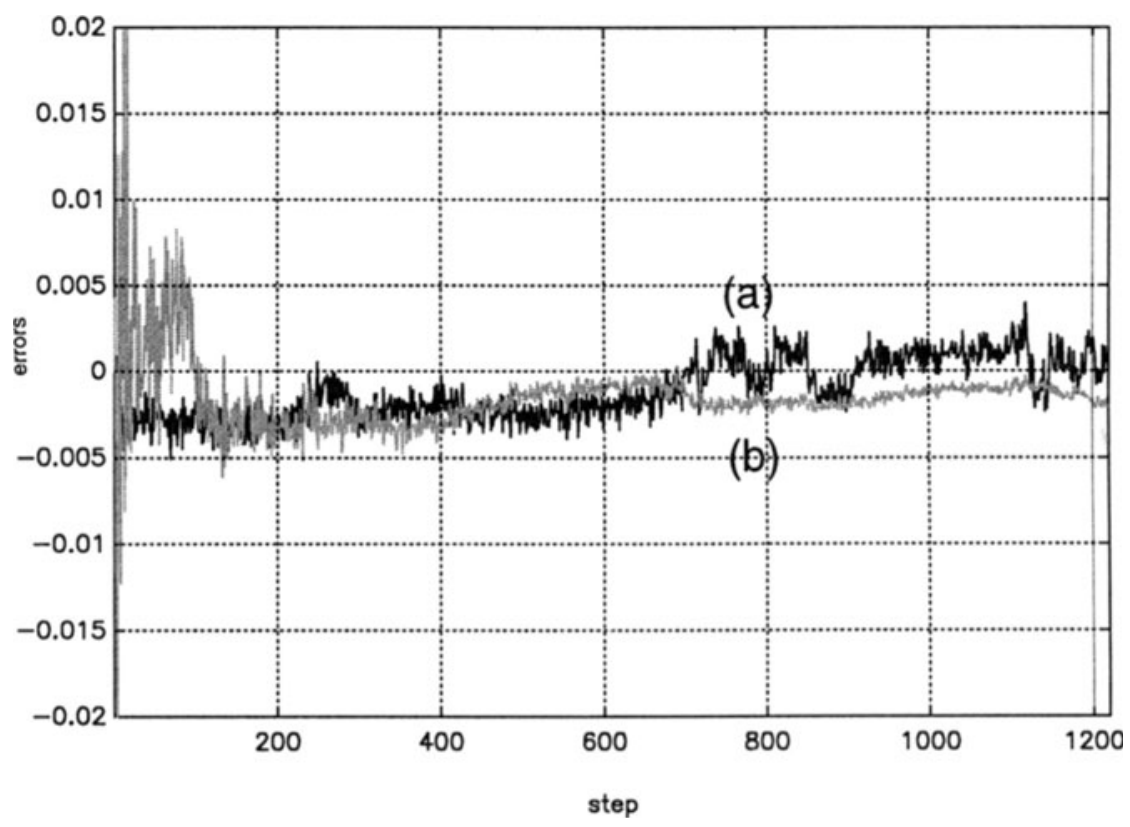

Fig. 26 Continuous transmision AWGN channel- $E_{b} / N_{0}=8 d B$ differences between min-survivor and per-survivor learning curves: (a)LMS $\mu=1 / 320$; (b) RLS $w=0.9999$

\subsubsection{AWGN channel with Doppler effect}

The learning curves for some min-survivor LMS and RLS receivers are presented in fig. 27. It is noteworthy that the RLS $\mathrm{w}=0.9999$ and LMS $\mu=1 / 160$, after initial convergence, are not able to follow the progressive rotation since they keep a very heavy track of past estimation history. RLS $\mathrm{w}=0.9999$, which is optimal in the AWGN channel case, diverges very fast since its estimation forgets practically nothing. RLS $\mathrm{w}=0.96$ and LMS $\mu=1 / 20$ learning curves behave similarly each other and fluctuate in dependence of the noise. However some reduction of estimation error with respect to start-up error is maintained. Fig. 28 shows the difference between min-survivor and per-survivor LMS and RLS optimal learning curves. Also in this case the per-survivor learning curves are referred to the estimation path associated to the ML sequence. The average value of the represented curves evaluates $1.2 \times 10^{-3}$ for LMS $\mu=1 / 20$ and $1.1 \times 10^{-3}$ for RLS $w=1 / 0.96$. As in the AWGN case, no different learning 
behavior is found between minsurvivor and persurvivor estimation techniques.

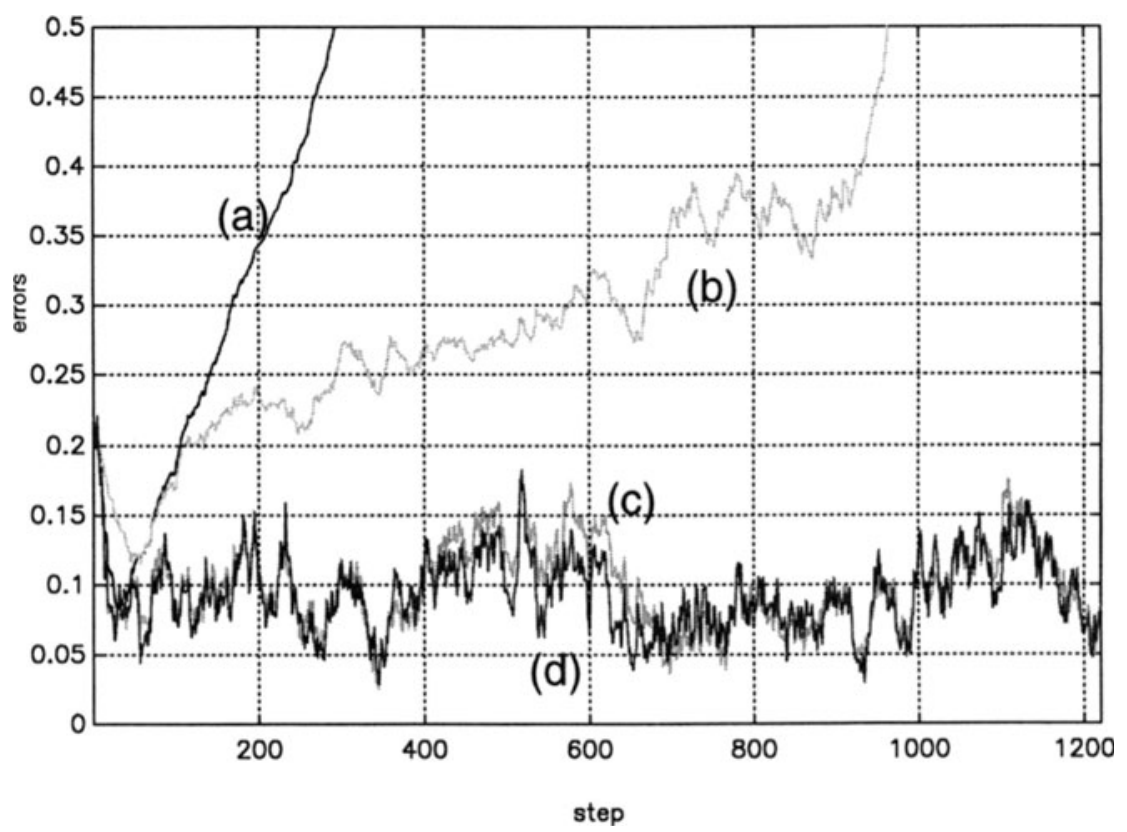

Fig. 27 Continuous transmision AWGN channel with Doppler effect$E_{b} / N_{0}=8 d B$ min-survivor learning curves: (a)RLS $\mathrm{w}=0.9999$; (b) LMS $\mu=1 / 80$; (c) LMS $\mu=1 / 20$; (d) RLS $\mathrm{w}=0.96$.

\section{Conclusions and open questions}

In this paper, topics about a digital euclidean distance polyphase MLSE structure has been discussed. The proposed structure performance has been shown to be, according with theory, insensitive to particular choice of carrier-phase and symbol-timing synchronization. Second, data-aided adaptive realizations of the euclidean distance MLSE receiver have been defined. Their performance has been studied in particular for the ETSI/GSM TDMA format in AWGN, Doppler and fading channel environments. All the obtained results show a performance superior to traditional receivers based only on midamble training. The simulations for continuous modulation format in the AWGN channel 


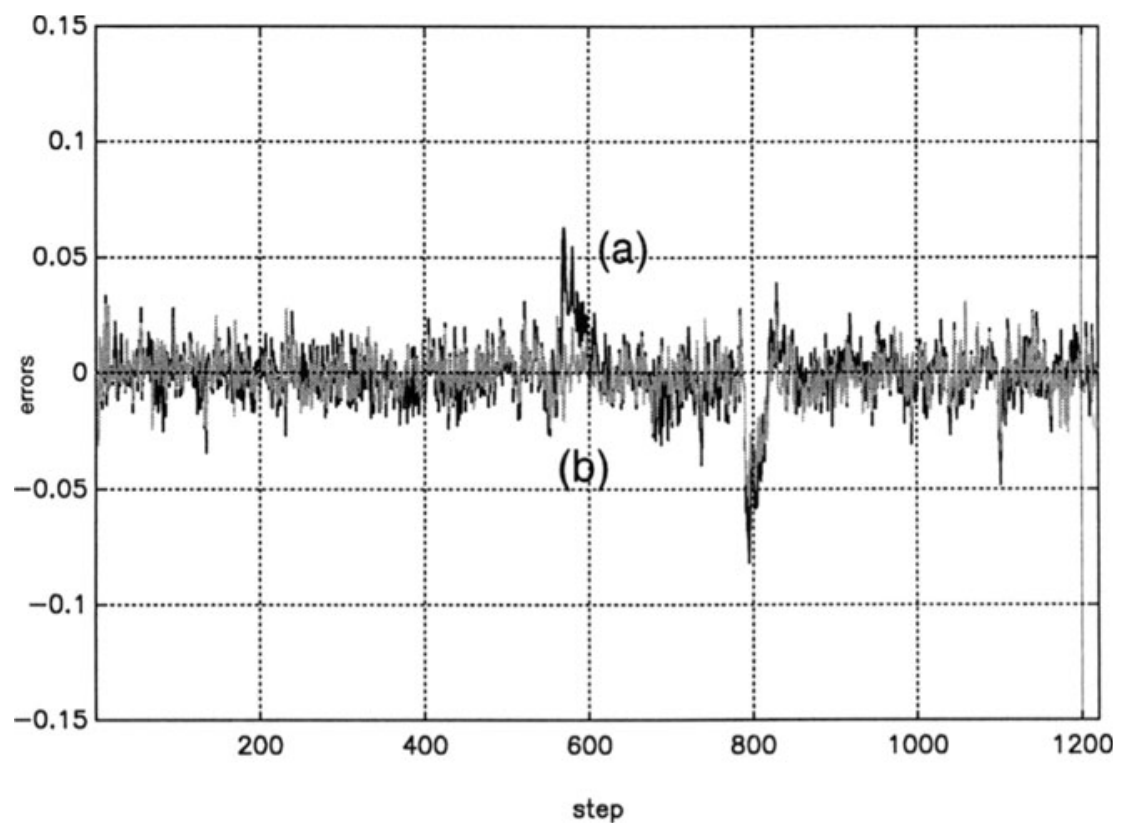

Fig. 28 Continuous transmission AWGN channel with Doppler effect$E_{b} / N_{0}=8 d B$ difference between min-survivor and per-survivor learning curves: (a)LMS $\mu=1 / 20$; (b) RLS $w=0.96$

environment have shown that the convergence time constants for adaptive techniques are somehow larger than the ETSI/GSM time burst duration, adaptive techniques can change the receiver performance enough to attain significant BER improvement in the ETSI/GSM format case as well. Among the adaptive realizations, the different LMS/RLS minsurvivor and persurvivor estimation techniques have all exhibited very similar performance. Consequently, the simplest structure, which is the proposed LMS min-survivor receiver can be regarded with particular interest.

Hence, in view of possible further simplification, open questions are left about:

- analysis of tracking algorithm and adaptive receivers performance varying the number $\mathrm{L}$ of estimated coefficients.

- performance evaluation of the analyzed adaptive receivers for the new ATDMA (Advanced Time Division multiple access) systems of third generation in the scenario of terrestrial and satellite integrated networks. 


\section{REFERENCES}

[1] C.-E. Sundberg, "Continuous Phase Modulation," IEEE Communications Magazine, Vol. 24, No. 4, pp.25-38, April 1986.

[2] P. A. Laurent, "Exact and approximate construction of digital phase modulation by superimposition of Amplitude Modulated Pulses (AMP)," IEEE Trans. Comm., Vol. COM-34, February 1986

[3] J. G. Proakis, Digital Communications, New York: McGraw-Hill, 1989.

[4] G. D. Forney, "Maximum-Likelihood Sequence Estimation of Digital Sequences in the Presence of Intersymbol Interference," IEEE Trans. Inform. Theory, Vol. IT-18, No. 3, May 1972.

[5] G. Ungerboeck, "Adaptive Maximum-Likelihood Receiver for CarrierModulated Data Transmission Systems," IEEE Trans. on Commun., Vol. 22, No. 5, May 1974.

[6] J. G. Proakis, "Adaptive Equalization for TDMA Digital Mobile Radio," IEEE Trans. Veh. Technol., Vol. 40, No 2, May 1991.

[7] F.R.Magee, J.G.Proakis, "Adaptive maximum-Likelihood Sequence Estimation for Digital Signaling in teh presence of Intersymbol Interference" IEEE Trans. on information Theory,January 1973

[8] R. Raheli, A. Polydoros, C.-K. Tzou "The principle of per-survivor processing: a general approach to approximate and adaptive MLSE," GLOBECOM '91, Phoenix (USA), Dec.1991.

[9] K. Murota, K. Hirade, "GMSK modulation for digital mobile telephony," IEEE Trans. Comm., Vol. 29, No. 7, July 1981.

[10] A. Baier, "Derotation Techniques in Receivers for MSK-type CPM Signals," Signal Processing V:theories and application. Elsevier Science Publishers 1990.

[11] W. R. Braun, U. Dersch, "A physical mobile radio channel model," IEEE Trans. Veh. Technol., Vol. 40, No. 2, May 1991.

[12] G.A.Arredondo, W.H. Chriss, E.H.Walker," A Multipath Fading Simulator for Mobile Radio,"IEEE Trans. Comm.,Vol. COM-21,No. 11,November 1973.

[13] A.Akki,F. Haber "A statistical Model of Mobile to Mobile Land Communication Channel", IEEE Trans. on Vehicular Technology,vol35,N.1,February 1986.

[14] GSM recommendation 05.05 (version 3.7.0).

[15] D. Messerschmitt, E. Lee," Digital Communications," Boston: Kluwer Academic Publisher, 1988.

[16] R. D'Avella, L. Moreno, M. Sant'Agostino, "An adaptive MLSE receiver for TDMA digital mobile radio," IEEE J. Select. Areas Commun., Vol. 7, No. 1, January 1989.

[17] K. Fukawa, H. Suzuki, "Adaptive equalization with RLS-MLSE for frequency-selective fast fading mobile radio channels," GLOBECOM'91, Phoenis (USA),Dec.1991. 
[18] E. Del Re, "Adaptive channel estimation for mobile radio," Proc. of COST 229 Workshop on adaptive Algorithms: application and non classical schemes, Vigo (Spain) March 1991

[19] E. Del Re, G. Castellini, L. Pierucci, F. Conti, "A within burst adaptive MLSE receiver for mobile TDMA cellular systems," IEEE ICASSP '92, San Francisco, March 1992.

[20] E. Del Re, G. Castellini, L. Pierucci, F. Conti, "An improved adaptive MLSE receiver for fading communications channels," EURASIP EUSIPCO '92, Brussells, August 1992.

[21] R.A. Ziegler, J.M. Cioff, "Estimation of timevarying digital mobile radio channels," GLOBECOM '91, Phoenix (USA),Dec.1991.

[22] B. Widrow, S. D. Stearns, "Adaptive signal processing," Prentice-Hall, Inc.: Englewood Cliffs 1985.

[23] J.R. Zeidler, "Performance analysis of LMS adaptive prediction filters," Proc. IEEE, Vol. 78, December 1990.

[24] G.D. Forney," Review of random tree codes," NASA Amer. Res. Cen., Moffett Field, Calif., Contract NAS2-3637, NASA CR 73176, Final Rep., December 1967.

[25] A.J. Viterbi," Convolutional codes and their performance in communication systems," IEEE Trans. Comm. Technol., Vol. COM-19, October 1971

[26] S.N. Crozier, D.D. Falconer, S.A. Mahmoud," Least sum of squared errors (LSSE) channel estimation," IEE Proc.-F, Vol. 138, No. 4, August 1991.

[27] J. H. Lodge, M. L. Moher, "Maximum likelihood sequence estimation of CPM signals transmitted over Rayleigh flat-fading channels," IEEE Trans. Comm., Vol. 38, No. 6, June 1990.

[28] N.Seshadri "Joint data detection and channel estimation using fast blind trellis search techniques" GLOBECOM 90 Conf. pp.1659-1663 Dec. 1990.

[29] H.Kubo,K.Murakami T.Fujino "An adaptive maximum likelihood sequence estimator for fast time-varying intersymbol interference channels" IEEE Trans. on Commun. Vol.42 N.2/3/4 February/March/April 1994.

[30] S. Haykin "Adaptive filter theory " Prentice- Hall International Editions USA 1991. 